\title{
La théorie des équations différentielles $p$-adiques et le Théorème de la monodromie $p$-adique
}

Zoghman Mebkhout

\begin{abstract}
In this lecture we introduce the reader to the proof of the $p$-adic monodromy theorem linking the $p$-adic differential equations theory and the local Galois $p$-adic representations theory.
\end{abstract}

\section{Introduction}

Cet exposé est motivé par la démonstration en mai $2001\left[\mathrm{M}_{2}\right]$ du théorème de la monodromie $p$-adique soulevé par J .M. Fontaine $\left[\mathrm{F}_{2}\right]$, établissant un lien hautement non trivial entre la théorie des équations différentielles $p$-adiques et la théorie des représentations galoisiennes locale $p$-adiques qui devrait avoir de nombreuses retombées. En particulier on obtient un exemple où le calcul différentiel a prise sur des questions concernant des groupes de Galois locaux de corps de nombres.

La théorie des $(\varphi, \Gamma)$-modules de Fontaine $\left[\mathrm{F}_{2}\right]$ classifiant les représentations galoisiennes locales $p$-adiques ramène le théorème de la monodromie $p$-adique au théorème de décomposition d'un module différentiel en modules de rang un dans le cas de l'existence d'une structure de Frobenius.

Pour la commodité du lecteur nous rappelons le théorème de la monodromie complexe et le théorème de la monodromie $\ell$-adique de A. Grothendieck qui ont joué un rôle très important dans les années 1970. Tous deux sont

2000 Mathematics Subject Classification : 12H25, 11F80, 14F, $14 \mathrm{~F} 30$.

Keywords : Équations différentielles $p$-adiques, monodromies, représentations galoisiennes $p$-adiques, corps des périodes $p$-adiques, $(\varphi, \Gamma)$-modules, exposants $p$-adiques, polygone de Newton $p$-adique. 
apparus dans la correspondance Grothendieck-Serre de l'année 1964 [G-S]. Les premières démonstrations sont dues à Grothendieck qui a introduit à cette occasion la théorie des cycles évanescents ([G-S], [SGA $\left.7_{I}\right]$ ).

D'autre part la théorie des représentations galoisiennes locales $p$-adiques de Fontaine est motivée par le problème du foncteur mystérieux posé par Grothendieck au congrès de Nice $[\mathrm{G}]$ reliant la cohomologie étale $p$-adique et la cohomologie de de Rham d'une variété propre et lisse sur un corps p-adique local.

La théorie des équations différentielles $p$-adiques a été motivée par le théorème de l'indice. Celui-ci est à la base de la définition de la catégorie des coefficients $p$-adiques et de ses propriétés de finitude, parallèle à la catégorie des coefficients $\ell$-adiques. Il permet de démontrer la rationalité des fonctions $L$ des fibrés $p$-adiques sur les courbes sur les corps finis et la factorisation polynomiale $p$-adique de la fonction Zêta d'une variété affine non singulière sur un corps fini donc, en particulier, sa rationalité.

Avant la démonstration du théorème de la monodromie $p$-adique, la théorie des représentations galoisiennes $p$-adiques et la théorie des équations différentielles $p$-adiques avaient des motivations bien distinctes et ont été développées de façon largement indépendante. Leur méthodes différent sensiblement.

Cependant on savait depuis longtemps que les propriétés de la ramification des corps locaux étaient similaires aux propriétés de l'irrégularité des équations différentielles. En fait la théorie du polygone de Newton $p$-adique d'une équation différentielle apparaît comme un intermédiaire entre la théorie de la ramification $\ell$-adique des représentations galoisiennes locales en égales caractéristiques $p>0$ et la théorie de l'irrégularité en caractéristique nulle.

Cet exposé peut servir au lecteur d'introduction à la fois au théorème de la monodromie dans les différents contextes et à la théorie des équations différentielles $p$-adiques. Pour les démonstrations complètes nous renvoyons le lecteur aux mémoires originaux cités dans la bibliographie nécessairement plus difficiles.

A l'occasion du Colloque en l'honneur de J. L. Vicente nous voudrions remercier tous les membres du département d'algèbre de l'Université de Seville pour les relations cordiales et amicales que nous avons eues depuis si longtemps. Nous espérons qu'elles ont contribué à la promotion des mathématiques qui est notre passion commune. 


\section{Le théorème de la monodromie complexe}

\subsection{Cas d'un morphisme analytique propre}

Soit $f$ une fonction propre

$$
f: X \rightarrow D
$$

d'une variété analytique complexe $X$ sur un petit disque $D$ voisinage de l'origine dans le plan complexe. On suppose de plus que $f$ est lisse en dehors de l'origine. Alors la restriction de $f$ au disque épointé $D^{*}$ est une fibration topologique en vertu du théorème d'Ereshman. Les images directes supérieures $R^{i} f_{*} \mathbb{C}_{X^{*}}$ forment des systèmes locaux d'espaces vectoriels complexes sur le disque épointé $D^{*}$. La fibre du système local $R^{i} f_{*} \mathbb{C}_{X^{*}}$ en un point $t$ s'identifie à la cohomologie complexe de la fibre $H^{i}\left(X_{t}, \mathbb{C}\right)$ qui est un espace vectoriel de dimension finie. On obtient comme cela les représentations de monodromie

$$
\rho: \Pi\left(D^{*}, t\right) \simeq \mathbb{Z} \rightarrow G L\left(H^{i}\left(X_{t}, \mathbb{C}\right)\right) .
$$

La monodromie est l'action $T:=\rho(1)$ du générateur qui est le lacet d'origine $t$ tournant dans le sens trigonométrique autour de l'origine.

Théorème 2.1.1 La représentation $\rho$ précédente est quasi-unipotente d'indice de nilpotence borné par $i+1$ : il existe un entier a tel que $\left(T^{a}-I d\right)^{i+1}=0$.

Démonstration. La démonstration originale de Grothendieck du théorème précédent ([G-S], [SGA $\left.7_{I}\right]$ ) utilise le théorème de la résolution des singularités d'Hironaka. En vertu de ce théorème on peut supposer que la fibre spéciale $Y:=f^{-1}(0)$ est un diviseur à croisements normaux. Notons $\tilde{D}^{*} \rightarrow D^{*}$ un revêtement universel du disque épointé et $\tilde{X}^{*} \rightarrow D^{*}$ le morphisme obtenu par ce changement de base. Fixons les notations à l'aide du diagramme suivant :

$$
\begin{array}{cccccc}
\tilde{X}^{*} & \rightarrow & X^{*} & \rightarrow & X & \leftarrow \\
\downarrow & & \downarrow & & \downarrow f & \\
\tilde{D}^{*} & \rightarrow & D^{*} & \rightarrow & D & \leftarrow
\end{array}
$$

Le complexe des cycles évanescents est défini par :

$$
\mathbf{R} \Psi_{f}\left(\mathbb{C}_{X}\right):=i^{-1} \mathbf{R} p_{*} \mathbb{C}_{\tilde{X}^{*}}
$$

où $i$ désigne l'inclusion de la fibre spéciale dans $X$ et $p$ l'application de $\tilde{X}^{*}$ dans $X$. Le complexe des cycles évanescents est muni d'une action de la monodromie. Le théorème de changement de bases pour un morphisme propre montre que l'on a un isomorphisme canonique

$$
\mathbf{R} f_{*} \mathbf{R} \Psi_{f}\left(\mathbb{C}_{X}\right) \simeq \mathbf{R} \Psi_{I d}\left(\mathbf{R} f_{*} \mathbb{C}_{X}\right)
$$


équivariant. D'où l'on déduit l'isomorphisme équivariant :

$$
H^{i}\left(Y, \mathbf{R} \Psi_{f}\left(\mathbb{C}_{X}\right)\right) \simeq H^{i}\left(X_{t}, \mathbb{C}\right)
$$

qui donne la suite spectrale équivariante :

$$
H^{p}\left(Y, R^{q} \Psi_{f}\left(\mathbb{C}_{X}\right)\right) \Longrightarrow H^{i}\left(X_{t}, \mathbb{C}\right) .
$$

Pour montrer le théorème il suffit de montrer qu'il existe un entier $a$ tel que l'action de $T^{a}-I d$ est nulle sur le faisceau

$$
R^{q} \Psi_{f}\left(\mathbb{C}_{X}\right):=i^{-1} R^{q} p_{*} \mathbb{C}_{\tilde{X}^{*}}
$$

pour tout $q \geq 0$. En effet cela entraîne que l'action de $T^{a}-I d$ est nulle sur les modules gradués de la filtration de longueur bornée par $i+1$ de la suite spectrale. Il suffit de montrer cette propriété localement sur le fibre spéciale $Y$ puisqu'elle est compacte. Pour un système convenable de coordonnées locales $x_{1}, \ldots, x_{n}$ au voisinage d'un point 0 la fonction $f$ s'écrit $f(x)=x_{1}^{k_{1}} \cdots x_{r}^{k_{r}}$ pour un entier $r \leq n$. La fibre du faisceau $R^{q} \Psi_{f}\left(\mathbb{C}_{X}\right)$ au point 0 s'identifie à la cohomologie de l'hypersurface $Z$ de l'ouvert $U^{n-r} \times U^{* r} \times \tilde{D}^{*}$, pour un petit voisinage $U$ de 0 , définie par l'équation

$$
x_{1}^{k_{1}} \cdots x_{r}^{k_{r}}=\exp (2 \pi \sqrt{-1} s)
$$

munie de l'action de la monodromie sur le dernier facteur. Soit $d$ le plus grand diviseur commun des entiers $k_{1}, \ldots, k_{n}$, alors l'hypersurface $Z$ précédente est la réunion disjointe des hypersurfaces $Z_{\ell}$ définies par

$$
x_{1}^{k_{1}^{\prime}} \cdots x_{r}^{k_{r}^{\prime}}=\xi^{l} \exp (2 \pi \sqrt{-1} s / d)
$$

pour $\ell=0, \ldots, d-1, \xi=\exp (2 \pi \sqrt{-1} / d), k_{1}=d k_{1}^{\prime}, \ldots, k_{r}=d k_{r}^{\prime}$ et l'action de la monodromie permute ces hypersurfaces. Chaque hypersurface $Z_{l}$ est stable par l'action de $T^{d}$ et nous allons voir que l'application $T^{d}$ est homotope à l'identité et donc opère trivialement sur la cohomologie. tion

Soit $\sum_{i=1, r} q_{i} k_{i}^{\prime}=1$ l'identité de Bézout et $F: Z_{l} \times[0,1] \rightarrow Z_{l}$ l'applica-

$$
\begin{gathered}
F\left(x_{1}, \ldots, x_{r}, x_{r+1}, \ldots, x_{n}, s, u\right):= \\
\left(x_{1} \exp \left(2 \pi \sqrt{-1} q_{1} u\right), \ldots, x_{r} \exp \left(2 \pi \sqrt{-1} q_{r} u\right), x_{r+1}, \ldots, x_{n}, s+d u\right) .
\end{gathered}
$$

On a

$$
F\left(x_{1}, \ldots, x_{r}, x_{r+1}, \ldots, x_{n}, s, 0\right)=I d \text { et } F\left(x_{1}, \ldots, x_{r}, x_{r+1}, \ldots, x_{n}, s, 1\right)=T^{d}
$$

et donc $F$ réalise une homotopie entre l'application identique et $T^{d}$. 


\subsection{Cas d'un morphisme local}

Soit $f:\left(\mathbb{C}^{n}, 0\right) \rightarrow(\mathbb{C}, 0)$ un germe de fonction analytique. Le théorème de fibration de Milnor [Mi] remplace le théorème d'Ereshman : pour une boule $B_{\epsilon}$ de rayon $\epsilon$ assez petit centrée à l'origine et pour un disque $D_{\eta}$ de rayon $\eta$ assez petit par rapport $\epsilon$ la fonction $f$ induit une fibration

$$
f: B_{\epsilon}^{*} \rightarrow D_{\eta}^{*}
$$

qui donne naissance aux systèmes locaux $R^{i} f_{*} \mathbb{C}_{B_{\epsilon}^{*}}$ sur le disque épointé et l'on a encore un isomorphisme de changement de bases

$$
\mathbf{R} f_{*} \mathbf{R} \Psi_{f}\left(\mathbb{C}_{B_{\epsilon}^{*}}\right) \simeq \mathbf{R} \Psi_{I d}\left(\mathbf{R} f_{*} \mathbb{C}_{B_{\epsilon}^{*}}\right)
$$

équivariant. Si l'on note encore $T$ l'action de la monodromie sur la fibre de Milnor on a encore :

Théorème 2.2.1 L'action de la monodromie sur la cohomologie de degré $i$ de la fibre de Milnor est quasi-unipotente d'indice de nilpotence borné par $i+1$ : il existe un entier a tel que $\left(T^{a}-I d\right)^{i+1}=0$.

Démonstration. En utilisant le théorème de résolution des singularités d'Hironaka la démonstration est alors identique à celle du cas propre. Mais Lê D. T. [L] a donné une démonstration géométrique du théorème précédent par la méthode des carousels qui est indépendante du théorème de la résolution des singularités et qui est très instructive.

D'autre part B. Malgrange a mis en évidence que le théorème de la monodromie locale est équivalent à la rationalité des zéros du polynôme de Bernstein-Sato attaché à $f\left(\left[\mathrm{Ma}_{1}\right],\left[\mathrm{Ma}_{2}\right]\right)$.

\subsection{Cas d'un morphisme algébrique propre}

Soit $f$ un morphisme propre et lisse

$$
f: X \rightarrow S
$$

de variétés algébriques non singulières sur un corps de caractéristique nulle tel que la base $S$ est une courbe affine. Notons $D R_{f}\left(\mathcal{O}_{X}\right)$ le complexe de de Rham relatif.

$$
0 \rightarrow O_{X} \rightarrow \Omega_{X / S}^{1} \rightarrow \cdots \rightarrow \Omega_{X / S}^{\operatorname{dim} X / S} \rightarrow 0
$$

Les images directes supérieures

$$
R^{i} f_{*} D R_{f}\left(\mathcal{O}_{X}\right)
$$

sont des fibrés algébriques sur $S$ munis d'une connexion canonique dite de Gauss-Manin. 
En chaque point à l'infini de $S$ un fibré à connexion donne naissance à un espace vectoriel de dimension finie sur un corps des séries formelles muni d'une connexion. Si ce point est régulier, en fixant une uniformisante, il existe une base dans laquelle la matrice de la connexion est constante à coefficients dans le corps résiduel. Soit $C$ une telle matrice de décomposition de Jordan $C=D+N,[D, N]=0$ telle que la matrice $D$ est diagonale et la matrice $N$ est nilpotente. Les classes modulo $\mathbb{Z}$ des valeurs propres de la matrice $D$ sont par définition les exposants locaux, ce sont des invariants du fibré. On dit que le fibré est quasi-unipotent d'indice de nilpotence borné par $i$ si les exposants sont des nombres rationnels et si $N^{i}=0$. C'est aussi un propriété indépendante de la base choisie.

Théorème 2.3.1 En un point à l'infini de la courbe $S$, pour tout $i \geq 0$ les fibrés à connexion $R^{i} f_{*} D R_{f}\left(\mathcal{O}_{X}\right)$ sont réguliers quasi-unipotents d'indice de nilpotence borné par $i+1$.

La très belle démonstration arithmétique de N. Katz $[\mathrm{K}]$ consiste d'abord à démontrer la nilpotence de la connexion de Gauss-Manin en caractéristique $p>0$ et à utiliser le théorème de décomposition de Turrittin pour en déduire la régularité et la quasi-unipotence d'indice borné par $i+1$. Elle est naturellement beaucoup plus simple et plus instructive que l'utilisation du théorème de résolution des singularités.

\section{Le théorème de la monodromie $\ell$-adique}

Soit un corps $K$ complet pour une valuation discrète d'anneau des entiers $\mathcal{O}_{K}$ et de corps résiduel $k$ de caractéristique $p>0$. Soit $X \rightarrow \operatorname{Spec} \mathcal{O}_{K}$ un morphisme de schémas de type fini et séparé. La fibre générique $X_{K}$ est une variété algébrique sur $K$ et la fibre spéciale $X_{k}$ est une variété algébrique sur $k$. Soit $K^{\text {sep }}$ une clôture séparable de $K$ et $G_{K}:=\operatorname{Gal}\left(K^{\text {sep }} / K\right)$ le groupe de Galois. Si $\ell$ est un nombre premier le groupe $G_{K}$ opère continûment par transport de structures sur la cohomologie étale $\ell$-adique $H_{e t}^{i}\left(X_{\bar{K}}, \mathbb{Q}_{\ell}\right)$ de la fibre générique géométrique. On obtient des représentations $\ell$-adiques particulièrement intéressantes :

$$
\rho: G_{K} \rightarrow G L\left(H_{e t}^{i}\left(X_{\bar{K}}, \mathbb{Q}_{\ell}\right)\right) .
$$

Le groupe d'inertie $I$ est défini par la suite exacte :

$$
1 \rightarrow I \rightarrow G_{K} \rightarrow G_{k} \rightarrow 1
$$

c'est un groupe topologique profini qui admet le dévissage :

$$
1 \rightarrow P \rightarrow I \rightarrow \prod_{l \neq p} \mathbb{Z}_{l}(1) \rightarrow 1
$$


où $\mathbb{Z}_{\ell}(1):=\lim _{\leftarrow h} \mu_{\ell^{h}}\left(k^{s e p}\right)$ est le pro- $\ell$-groupe des racines de l'unité d'ordre une puissance de $l$ contenu dans une clôture séparable $k^{s e p}$ du corps résiduel qui est naturellement un $G_{k}$-module. L'action est donnée par $\sigma(\xi):=\xi^{\chi(\sigma)}$ où $\chi: G_{k} \rightarrow \mathbb{Z}_{\ell}^{*}$ est le caractère cyclotomique.

Théorème 3.0.2 Supposons que $\ell$ est distinct de $p$ alors les représentations $\ell$-adiques $H_{e t}^{i}\left(X_{\bar{K}}, \mathbb{Q}_{l}\right)$ du groupe de galois $G_{K}$ sont quasi-unipotentes : un sous-groupe ouvert I' du groupe d'inertie opère de façon unipotente : l'endomorphisme $\rho(\sigma)-I d$ est nilpotent pour tout $\sigma \in I^{\prime}$.

$\mathrm{Si}$ on disposait du théorème de la résolution des singularités dans ce contexte on pourrait reproduire la démonstration du cas complexe pour obtenir en plus la borne $i+1$ comme indice de nilpotence. C'est ce qu'a fait d'ailleurs Grothendieck dans le cas d'une surface arithmétique en utilisant le théorème d'Abhyankar dans sa lettre à Serre datée du 30 octobre 1964 [G-S]. Mais heureusement pour la théorie $\ell$-adique, Grothendieck a montré ce théorème dans [SGA $7_{I}$ ] comme cas particulier du théorème suivant nettement plus simple et indépendant de toute résolution des singularités :

Théorème 3.0.3 Supposons que $\ell$ est distinct de $p$ et qu'aucune extension finie de $k$ ne contient toutes les racines de l'unité d'ordre une puissance de $\ell$, alors toute représentation $\ell$-adique continue $\rho: G_{K} \rightarrow G L(E)$ du groupe de galois $G_{K}$ est quasi-unipotente : un sous-groupe ouvert $I^{\prime}$ du groupe d'inertie opère de façon unipotente : l'endomorphisme $\rho(\sigma)-I d$ est nilpotent pour tout $\sigma \in I^{\prime}$.

Démonstration. Nous reprenons pour la commodité du lecteur les arguments de l'appendice [S-T]. Le groupe $G_{K}$ étant compact son image $\rho\left(G_{K}\right)$ est compacte dans le groupe $G L(E)$ et donc $E$ admet un réseau $E^{\prime}$ sur $\mathbb{Z}_{\ell}$ qui est stable par l'action de $G_{K}$. Soit $I d+\ell E n d\left(E^{\prime}\right)$ le sous-groupe de $G L\left(E^{\prime}\right)$ des automorphismes qui opèrent trivialement sur la réduction modulo $\ell$ du réseau $E^{\prime}$. Le point est que le groupe $I d+\ell E n d\left(E^{\prime}\right)$ qui est la limite projective des $\ell$-groupes finis $I d+\ell E n d\left(E^{\prime}\right) / I d+(\ell)^{h} \operatorname{End}\left(E^{\prime}\right), h \geq 2$ est un pro- $\ell$-groupe ouvert d'indice fini dans $G L\left(E^{\prime}\right)$. Soit $I^{\prime}$ le sous-groupe

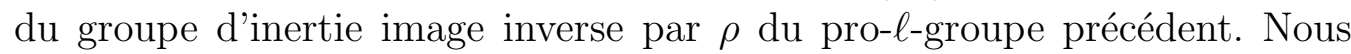
allons montrer que, pour tout $\sigma \in I^{\prime}, \rho(\sigma)$ est unipotent.

Le sous-groupe $I^{\prime}$ est groupe d'inertie du groupe de Galois $G_{K^{\prime}}$ d'une extension finie $K^{\prime}$ de $K$. Soit $K_{n r}^{\prime}$ l'extension maximale non ramifiée de $K^{\prime}$, c'est-à-dire l'extension dont le groupe de Galois absolu est le sous-groupe $I^{\prime}$ et $I_{\ell}^{\prime}$ le sous-groupe défini par la suite exacte

$$
1 \rightarrow I_{\ell}^{\prime} \rightarrow I^{\prime} \rightarrow \mathbb{Z}_{\ell}(1) \rightarrow 1
$$

Le groupe $I_{\ell}^{\prime}$ est un pro-groupe d'ordre premier à $\ell$ par construction. Son image $\rho\left(I_{\ell}^{\prime}\right)$ dans le pro- $\ell$-groupe $I d+\ell E n d\left(E^{\prime}\right)$ est triviale. 
Soit $K_{\ell}^{\prime}$ l'extension de $K_{n r}^{\prime}$ de groupe de Galois $I_{\ell}^{\prime}$ alors par construction l'action $\rho \mathrm{du}$ groupe $G_{K^{\prime}}$ se fait à travers le groupe de Galois $\operatorname{Gal}\left(K_{\ell}^{\prime} / K^{\prime}\right)$ qui s'insère dans la suite exacte :

$$
1 \rightarrow \operatorname{Gal}\left(K_{\ell}^{\prime} / K_{n r}^{\prime}\right) \rightarrow \operatorname{Gal}\left(K_{\ell}^{\prime} / K^{\prime}\right) \rightarrow \operatorname{Gal}\left(k^{\prime s e p} / k^{\prime}\right) \rightarrow 1,
$$

et par construction on a un isomorphisme canonique :

$$
\mathbb{Z}_{\ell}(1) \simeq \operatorname{Gal}\left(K_{\ell}^{\prime} / K_{n r}^{\prime}\right)
$$

Le groupe $\operatorname{Gal}\left(K_{\ell}^{\prime} / K_{n r}^{\prime}\right)$ est muni d'une action de $G_{k^{\prime}}$ par conjugaison après relèvement à $\operatorname{Gal}\left(K_{\ell}^{\prime} / K^{\prime}\right)$. On vérifie que l'isomorphisme (*) est équivariant par l'action du groupe de Galois $G_{k^{\prime}}$. Si $\sigma$ est un élément de $\operatorname{Gal}\left(K_{\ell}^{\prime} / K_{n r}^{\prime}\right)$, pour tout élément $t$ de $G_{k^{\prime}} \sigma$ et $\sigma^{\chi(t)}$ sont conjugués dans $\operatorname{Gal}\left(K_{\ell}^{\prime} / K^{\prime}\right)$. Les endomorphismes

$$
\log (\rho(\sigma)) \text { et } \log \left(\rho(\sigma)^{\chi(t)}\right)=\chi(t) \log (\rho(\sigma))
$$

sont conjugués. Si $a_{i}$ désigne la $i$-ème fonction symétrique des racines du polynôme caractéristique de $\log (\rho(\sigma))$, on trouve les égalités

$$
a_{i}(x)=\chi(t)^{i} a_{i}(x)
$$

pour tout $t$ dans $G_{k}$. La condition sur le corps résiduel montre qu'il existe un élément $t$ tel que $\chi(t)$ n'est pas racine de l'unité, ce qui entraîne que les fonctions $a_{i}$ sont nulles, $\log (\rho(\sigma))$ est nilpotent et son exponentielle $\rho(\sigma)$ est quasi-unipotente.

Le théorème de la monodromie $\ell$-adique est la principale raison du succès de la théorie $\ell$-adique qui n'a pas eu à affronter le problème de la résolution des singularités en caractéristique $p>0$.

\section{Le théorème de la monodromie $p$-adique}

La théorie des représentations $p$-adiques due à J. M. Fontaine et ses élèves a comme point de départ le problème du foncteur mystérieux posé par Grothendieck [G] dans le congrès de Nice de 1970.

Soient $p$ un nombre premier et $\mathbb{Q}_{p} \hookrightarrow K$ une extension complète à valuation discrète à corps résiduel parfait et $X / K$ une variété propre et lisse. On peut considérer la cohomologie de de Rham $H_{D R}^{\bullet}(X / K)$ qui est un $K$-espace de dimension finie muni de sa filtration de Hodge et la cohomologie étale $p$-adique $H_{e ́ t}^{\bullet}\left(X_{\bar{K}}, \mathbb{Q}_{p}\right)$ qui est un $\mathbb{Q}_{p}$-espace vectoriel de dimension finie muni d'une action continue du groupe de Galois absolu $G_{K}:=\operatorname{Gal}(\bar{K} / K)$ fournissant des exemples de représentations $p$-adiques particulièrement intéressantes. Par ailleurs comme on est en caractéristique nulle tous ces espaces fournissent les bons nombres de Betti de $X$ tout comme les espaces de cohomologie $\ell$-adiques pour $\ell \neq p$. 
Le problème suggéré par la théorie des motifs est de définir un isomorphisme fonctoriel de comparaison entre la cohomologie de de Rham $H_{D R}^{\bullet}(X / K)$ et la cohomologie étale $p$-adique $H_{e ́ t}^{\bullet}\left(X_{\bar{K}}, \mathbb{Q}_{p}\right)$.

Fontaine a réalisé le pas décisif $\left[\mathrm{F}_{1}\right]$ en introduisant le corps des périodes $p$-adiques $B_{d R}$ qui est un corps à valuation discrète extension du corps $K$ et muni d'une action continue du groupe de Galois $G_{K}$ et fait la conjecture $C_{d R}$ de $\left[\mathrm{F}_{1}\right]$. Cette conjecture est devenue le théorème suivant qui résout le problème du foncteur mystérieux :

Théorème 4.0.4 Il existe un isomorphisme de modules galoisiens fonctoriel en $X$ :

$$
B_{D R} \otimes_{\mathbb{Q}_{p}} H_{e ́ t}^{\bullet}\left(X_{\bar{K}}, \mathbb{Q}_{p}\right) \simeq B_{d R} \otimes_{K} H_{d R}^{\bullet}(X / K),
$$

où le groupe $G_{K}$ opère diagonalement sur le membre de gauche et seulement sur le corps $B_{d R}$ sur le membre de droite.

Après plusieurs cas particuliers de ce théorème démontrés par plusieurs auteurs Fontaine, Messing, Kato, Hyodo, Faltings ce théorème est démontré géométriquement par Tsuji $[\mathrm{T}]$.

\subsection{Catégories des représentations $p$-adiques}

Les conjectures de Fontaine $C_{c r i s}, C_{d R}, C_{s t}$ et $C_{p s t}$ sur la cohomologie $H_{\dot{e} t}^{\bullet}\left(X_{\bar{K}}, \mathbb{Q}_{p}\right)$ étale $p$-adique d'une variété propre et lisse ont servi de modèle à la théorie des représentations $p$-adiques générales $\left(\left[\mathrm{F}_{2}\right],\left[\mathrm{F}_{3}\right]\right)$.

Appelons représentation $p$-adique un homomorphisme continu

$$
\rho: G_{K} \rightarrow G L(E)
$$

du pro-groupe $G_{K}$ dans le groupe des automorphismes linéaires d'un espace vectoriel de dimension finie sur $\mathbb{Q}_{p}$ muni de la topologie $p$-adique. On note $\operatorname{Rep}\left(G_{K}\right)$ la catégorie des représentations $p$-adiques qui est de façon évidente

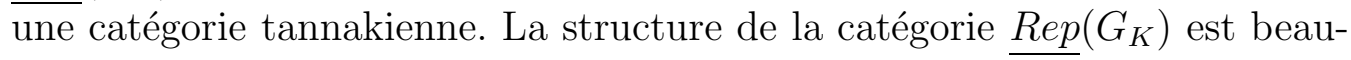
coup plus subtile que son analogue $\ell$-adique. La différence essentielle est que l'action du $p$-pro-groupe $P$ ne se fait pas à travers un quotient fini et la définition d'une représentation quasi-unipotente n'est pas claire. Pourtant Fontaine a introduit une hiérarchie entre ces représentations :

$$
\begin{aligned}
\text { cristalline } \Longrightarrow & \text { semi-stable } \Longrightarrow \text { potentiellement semi-stable } \Longrightarrow \text { de Rham } \\
& \Longrightarrow \text { Hodge-Tate } \Longrightarrow \text { représentation } p \text {-adique. }
\end{aligned}
$$

A chaque fois la cohomologie étale $p$-adique $H_{e ̂ t}^{\bullet}\left(X_{\bar{K}}, \mathbb{Q}_{p}\right)$ d'une variété propre et lisse sur $K$ fournit des exemples hautement non triviaux. Ainsi la cohomologie étale $p$-adique d'une variété propre et lisse admettant bonne réduction 
est cristalline, celle d'une variété admettant une réduction semi-stable est semi-stable, la cohomologie étale $p$-adique d'une variété propre et lisse est de de Rham. Il existe des représentations de Hodge-Tate qui ne sont pas de de Rham et des représentations $p$-adiques qui ne sont pas de Hodge-Tate. Tous ces théorèmes sont des résultats non triviaux dus à Fontaine $[\mathrm{F}]$, Faltings $[\mathrm{Fa}]$, Hyodo-Kato [H-K], Tsuji $[\mathrm{T}]$. Toutes les inclusions sont strictes à l'exception de la troisième.

Dans l'étude des représentations p-adiques Fontaine a dégagé deux problèmes importants.

Le premier, l'équivalence entre l'admissibilité et la faible admissibilité pour un $(\varphi, N)$-module filtré donne une description en terme de modules de la catégorie des représentions $p$-adique semi-stable. Il a été résolu par Fontaine-Colmez en 1999 [F-C] et nous n'en parlerons pas dans cet exposé.

Le second, la potentielle semi-stabilité pour une représentation $p$-adique de de Rham a été résolu en mai $2001\left[\mathrm{M}_{2}\right]$. C'est l'objet de cet exposé. Comme a priori la catégorie des représentations $p$-adiques de de Rham est plus grande que la catégorie des représentations $p$-adiques potentiellement semi-stables, il doit être plus facile de montrer qu'une représentation est de de Rham.

\subsection{Le corps des périodes $p$-adiques et la catégorie $\underline{\operatorname{Rep}}_{d R}\left(G_{K}\right)$ des représentations $p$-adiques de de Rham}

Nous allons définir la catégorie $\underline{R e p}_{d R}\left(G_{K}\right)$ des représentations p-adiques de de Rham. Pour cela il faut introduire le corps des périodes $p$-adiques $B_{d R}$. Soit $C$ le complété d'une clôture algébrique de $K$ et $\mathcal{O}_{C}$ l'anneau de ses entiers. Soit $\left(x=x^{(0)}, \ldots\right)$ l'ensemble $\tilde{E}^{+}$des suites d'éléments de l'anneaux $\mathcal{O}_{C}$ satisfaisant les relations $\left(x^{(n+1)}\right)^{p}=x^{(n)}$. On munit l'ensemble $\tilde{E}^{+}$ du produit $\left(x y=x^{(0)} y^{(0)}, \ldots\right)$ et de la somme $\left(x+y=z^{(0)}, \ldots\right)$ avec $z^{(m)}:=\lim _{n \rightarrow \infty}\left(x^{(m+n)}+y^{(m+n)}\right)^{p^{n}}$. C'est alors un anneau de caractéristique $p$ parfait. Son corps des fractions $\tilde{E}$ est algébriquement clos. On note $W\left(\tilde{E}^{+}\right)$ l'anneau des vecteurs de Witt à coefficients dans $\tilde{E}^{+}$. L'application qui à un vecteur de Witt $\left(x_{0}, x_{1}, \ldots\right)$ associe la limite quand $n \rightarrow \infty$ de

$$
x_{0}^{(n)} p^{p^{n}}+p x_{1}^{(n)} p^{n-1}+\cdots+p^{n} x_{n}^{(n)}
$$

est un homomorphisme surjectif d'anneau dans $\mathcal{O}_{C}$ :

$$
W\left(\tilde{E}^{+}\right) \rightarrow \mathcal{O}_{C}
$$

Son noyau est un idéal principal. Il en est de même de l'homomorphisme :

$$
\theta: W\left(\tilde{E}^{+}\right) \otimes_{W(k)} K \rightarrow C .
$$


On définit l'anneau $B_{d R}^{+}$par :

$$
B_{d R}^{+}:=\lim _{\leftarrow} W\left(\tilde{E}^{+}\right) \otimes_{W(k)} K /(\operatorname{Ker} \theta)^{n} .
$$

Le corps des fractions de l'anneau $B_{d R}^{+}$est par définition le corps $B_{d R}$ des périodes $p$-adiques. C'est une $K$-algèbre muni d'une action continue du groupe de Galois $G_{K}$. C'est aussi un corps à valuation discrète d'anneau des entiers $B_{d R}^{+}$et de corps résiduel $C$. On a un plongement naturel équivariant multiplicatif de $\mathbb{Z}_{p}(1)$ dans $\tilde{E}^{+}$. Si $x$ est un élément de $\tilde{E}^{+}$, on note $[x]:=(x, 0, \ldots)$ son représentant de Teichmller dans $W\left(\tilde{E}^{+}\right)$. Si $x$ est un élément de $\mathbb{Z}_{p}(1)$ la série :

$$
\log ([x]):=\sum_{n \geq 1}(-1)^{n-1}([x]-1)^{n} / n
$$

converge vers un élément de $B_{d R}^{+}$et on obtient un plongement $G_{K}$-équivariant additif de $\mathbb{Z}_{p}(1)$ dans $B_{d R}^{+}$. Pour un générateur $x$ de $\mathbb{Z}_{p}(1), \log ([x])$ est une uniformisante $t$ de $B_{d R}^{+}$et est l'analogue $p$-adique de $2 \pi \sqrt{-1}$.

Le corps $B_{d R}$ est isomorphe au corps des séries formelles $C((t))$ mais il n'existe pas d'isomorphisme équivariant pour l'action du groupe $G_{K}$, ce qui rend inutile cet isomorphisme.

Définition 4.2.1 On dit qu'une représentation p-adique $V$ de rang $m$ est de de Rham si elle est trivialisable sur le corps des périodes p-adiques $B_{d R}$ comme module galoisien sur le groupe $G_{K}$, autrement dit s'il existe un isomorphisme :

$$
B_{d R} \otimes_{\mathbb{Q}_{p}} V \simeq\left(B_{d R}\right)^{m}
$$

où le groupe $G_{K}$ opère diagonalement sur le membre de gauche et de façon naturelle sur le membre de droite.

La matrice dans une base de $V$ d'un isomorphisme est la matrice des périodes. Le théorème de comparaison montre que la cohomologie étale $p$-adique d'une variété propre et lisse sur $K$ est de de Rham.

\subsection{L'anneau $B_{s t}$ et la catégorie $\underline{R e p}_{p s t}\left(G_{K}\right)$ des représentations $p$-adiques potentiellement semi-stables}

Nous allons définir la catégorie $\underline{R e p}_{p s t}\left(G_{K}\right)$ des représentations $p$-adiques potentiellement semi-stables. Pour cela il faut introduire l'anneau $B_{\text {cris }}$ et l'anneau $B_{s t}$ qui est un anneau de polynôme sur l'anneau $B_{\text {cris }}$. Soit $\xi$ un générateur de l'idéal noyau de l'homomorphisme $\theta$. On considère la $W\left(\tilde{E}^{+}\right)$algèbre $W\left(\tilde{E}^{+}\right)[1 / p]$ et sa sous $W\left(\tilde{E}^{+}\right)$-algèbre $W\left(\tilde{E}^{+}\right)^{p d}$ engendrée par le 
générateur les puissances divisées $(\xi)^{n} / n$ ! et son complété $p$-adique $A_{\text {cris }}$. L'algèbre $A_{\text {cris }}$ qui est solution d'un problème universel ne dépend pas du générateur $\xi,\left[\mathrm{F}_{3}\right]$. Si $x$ est un élément de $\mathbb{Z}_{p}(1)$ la série :

$$
\log ([x]):=\sum_{n \geq 1}(-1)^{n-1}([x]-1)^{n} / n
$$

converge vers un élément de $A_{c r i s}$ et on obtient un plongement $G_{K}$-équivariant de $\mathbb{Z}_{p}(1)$ dans $A_{\text {cris }}$. On note $t$ l'image d'un générateur de $\mathbb{Z}_{p}(1)$. On pose :

$$
B_{\text {cris }}^{+}:=A_{\text {cris }}[1 / p], B_{\text {cris }}:=B_{\text {cris }}^{+}[1 / t] .
$$

Le morphisme de Frobenius $\varphi$ s'étend à $A_{\text {cris }}$ et l'on a $\varphi(t)=p t$ qui permet d'étendre l'action de Frobenius à l'anneau $B_{\text {cris }}$.

Définition 4.3.1 On dit qu'une représentation p-adique $V$ de rang $m$ est cristalline si elle est trivialisable sur l'anneau $B_{\text {cris }}$ comme module galoisien sur le groupe $G_{K}$, autrement dit s'il existe un isomorphisme :

$$
B_{\text {cris }} \otimes_{\mathbb{Q}_{p}} V \simeq\left(B_{\text {cris }}\right)^{m},
$$

où le groupe $G_{K}$ opère diagonalement sur le membre de gauche et de façon naturelle sur le membre de droite.

Soit $\left(x=x^{(0)}, \ldots\right)$ un élément de $\tilde{E}^{+}$tel que $x^{(0)}$ est une uniformisante de $K$. Alors $[x] / x^{(0)}$ appartient à $1+\operatorname{Ker}(\theta)$ de sorte que $u:=\log \left([x] / x^{(0)}\right)$ est un élément du corps $B_{d R}$. L'élément $u$ ne dépend que de l'uniformisante $x^{(0)}$ et est transcendant sur $B_{\text {cris }}\left[\mathrm{F}_{3}\right]$ de sorte que le sous-anneau $B_{s t}:=$ $B_{\text {cris }}[u]$ de $B_{d R}$ est un anneau de polynômes sur $B_{\text {cris }}$. L'anneau $B_{s t}$ est stable par l'action de $G_{K}$. On prolonge l'action du Frobenius à $B_{s t}$ en posant $\varphi(u):=p u$. On définit un opérateur de monodromie $N$ sur $B_{s t}$ comme l'unique $B_{\text {cris }}$-dérivation telle que $N(u):=1$.

Définition 4.3.2 On dit qu'une représentation p-adique $V$ de rang $m$ est semi-stable si elle est trivialisable sur l'anneau $B_{\text {st }}$ comme module galoisien sur le groupe $G_{K}$, autrement dit s'il existe un isomorphisme :

$$
B_{s t} \otimes_{\mathbb{Q}_{p}} V \simeq\left(B_{s t}\right)^{m}
$$

où le groupe $G_{K}$ opère diagonalement sur le membre de gauche et de façon naturelle sur le membre de droite. On dit qu'une représentation p-adique est potentiellement semi-stable si elle devient semi-stable après extension finie du corps de base $K$. 
On alors les inclusions

$$
\underline{\operatorname{Rep}}_{\text {cris }}\left(G_{K}\right) \subset \underline{\operatorname{Rep}}_{s t}\left(G_{K}\right) \subset \underline{\operatorname{Rep}}_{p s t}\left(G_{K}\right) \subset \underline{\operatorname{Rep}} d R\left(G_{K}\right) \subset \underline{\operatorname{Rep}}\left(G_{K}\right) .
$$

Le théorème de la monodromie $p$-adique conjecturé par Fontaine est :

Théorème 4.3.3 Soit une extension $\mathbb{Q}_{p} \rightarrow K$ à valuation discrète et à corps résiduel parfait alors l'inclusion

$$
\underline{\operatorname{Rep}}_{p s t}\left(G_{K}\right) \subset \underline{\operatorname{Rep}}_{d R}\left(G_{K}\right)
$$

de la catégorie des représentations potentiellement semi-stables dans la catégorie des représentations de de Rham est une égalité.

La théorie des $(\varphi, \Gamma)$-modules classifiant les représentations $p$-adiques ramène le théorème 4.3 .3 au théorème 6.3 .1 de décomposition en modules différentiels de rang 1.

\subsection{Théorie des $(\varphi, \Gamma)$-modules}

La théorie des $(\varphi, \Gamma)$-modules initiée par Fontaine dans son article du Grothendieck Festschrift II $\left[\mathrm{F}_{2}\right]$ remplace une représentation $p$-adique, qui un est objet délicat mais apparaît naturellement en géométrie arithmétique, par un module sur des anneaux de séries, objet plus concret qui permet de l'étudier sans perdre de l'information. Cette idée a abouti à faire le lien particulièrement fructueux entre la théorie des représentations $p$-adiques et la théorie des équations différentielles $p$-adiques. Il y a trois étapes faisant intervenir trois anneaux galoisiens.

\subsubsection{Les anneaux $\tilde{E}, \tilde{A}, \tilde{B}, E, A, B$}

Soient $k$ un corps parfait de caractéristique $p>0, W(k)$ l'anneau des vecteurs de Witt à coefficients dans $k, K_{0}$ son corps des fractions et $C$ le complété d'une clôture algébrique $\bar{K}_{0}$ de $K_{0}$. Si $K_{0, \infty}$ est l'extension cyclotomique maximale de $K_{0}$ la théorie de Galois fournit le dévissage

$$
0 \rightarrow H_{K_{0}} \rightarrow G_{K_{0}} \rightarrow \Gamma_{K_{0}} \rightarrow 0
$$

où $G_{K_{0}}:=\operatorname{Gal}\left(\bar{K}_{0} / K_{0}\right)$ et $H_{K_{0}}:=\operatorname{Gal}\left(\bar{K}_{0} / K_{0, \infty}\right)$. Le groupe $H_{K_{0}}$ apparaît aussi comme le noyau du caractère cyclotomique $\chi: G_{K_{0}} \rightarrow Z_{p}^{*}$ donnant l'action de $G_{K_{0}}$ sur les racines de l'unité d'ordre une puissance de $p$. Le groupe $G_{K_{0}}$ opère sur $C$ par continuité.

Le corps $\tilde{E}$ est un corps algébriquement clos de caractéristique $p$ complet pour la valuation définie par $v_{E}(x):=v_{p}\left(x^{(0)}\right)\left[\mathrm{F}_{2}\right]$. 
Soit $\epsilon:=\left(1, \epsilon^{(1)}, \ldots\right)$ un élément du corps $\tilde{E}$ tel que $\epsilon^{(1)} \neq 1$, autrement dit on extrait successivement une suite de racines $p$-ème de l'unité. On a $v_{E}(\epsilon-1)=p /(p-1)$ et on note $E_{k}$ le sous-corps $k((\epsilon-1))$ de $\tilde{E}$ et $E$ sa clôture séparable dans $\tilde{E}$. La théorie du corps des normes permet d'identifier canoniquement $\operatorname{Gal}\left(E / E_{k}\right)$ avec $H_{K_{0}}[\mathrm{~F}-\mathrm{W}]$. Autrement dit le groupe $H_{K_{0}}$ s'identifie au groupe de Galois d'une clôture séparable du corps des séries formelles à coefficients dans le corps $k$.

On note $\tilde{A}:=W(\tilde{E})$ l'anneau des vecteurs de Witt à coefficients dans $\tilde{E}$ et $\tilde{B}$ son corps des fractions. Le groupe de Galois $G_{K_{0}}$ opère sur $\tilde{E}, \tilde{A}, \tilde{B}$. L'élévation à la puissance $p$ induit le morphisme de Frobenius $\varphi$ sur $\tilde{E}$ et par fonctorialité sur $\tilde{A}$ et $\tilde{B}$.

Si $a$ est un élément de $\tilde{E}$ on note $[a]$ son représentant de Teichmller $[a]:=$ $(a, 0, \ldots$,$) dans \tilde{A}$ de sorte que tout élément $x$ de $\tilde{A}$ admet un développement unique $x=\sum_{i=0}^{\infty}\left(p^{i}\left[x_{i}\right]\right)$. Soit $\pi_{K_{0}}:=[\epsilon]-1$ l'élément de $\tilde{A}$ et $A_{K_{0}}$ le complété $p$-adique de l'anneau $W(k)\left[\pi_{K_{0}}, \frac{1}{\pi_{K_{0}}}\right]$. On trouve que :

$$
\varphi\left(\pi_{K_{0}}\right)=\left(1+\pi_{K_{0}}\right)^{p}-1, g\left(\pi_{K_{0}}\right)=\left(1+\pi_{K_{0}}\right)^{\chi(g)}-1, g \in G_{K_{0}} .
$$

L'anneau $A_{K_{0}}$ et son corps des fractions $B_{K_{0}}$ sont stables par le morphisme de Frobenius $\varphi$ et par l'action du groupe de Galois $G_{K_{0}}$.

Soit $\mathcal{E}_{K_{0}}$ l'anneau des séries de Laurent $\sum_{i \in \mathbb{Z}} a_{i} x^{i}$ en la variable $x$ à coefficients dans le corps $K_{0}$ telles que les coefficients sont uniformément bornés et la suite des termes négatifs tend vers zéro, alors l'application

$$
\mathcal{E}_{K_{0}} \rightarrow B_{K_{0}}
$$

qui a la série $\sum_{i \in \mathbb{Z}} a_{i} x^{i}$ associe la somme $\sum_{i \in \mathbb{Z}} a_{i} \pi_{K_{0}}^{i}$ est un isomorphisme d'anneaux ce qui donne une description très concrète de l'anneau $B_{K_{0}}$.

Soit $B$ l'adhérence dans $\tilde{B}$ de l'extension maximale non ramifiée de $B_{K_{0}}$ et $A:=B \cap \tilde{A}$. L'anneau $A$ est un anneau à valuation discrète complet de corps de fraction $B$ et de corps résiduel $E$.

Pour une extension finie $K$ de $K_{0}$ on pose :

$$
A_{K}:=A^{H_{K}}, B_{K}:=B^{H_{K}} .
$$

Les anneaux $A_{K}$ et $B_{K}$ se trouvent munis d'actions du morphisme de Frobenius et du groupe quotient $\Gamma_{K}$ qui commutent. Si $K$ est non ramifié le corps $B_{K}$ est encore canoniquement isomorphe au corps $\mathcal{E}_{K}$. Mais si $K$ est totalement ramifié c'est-à-dire admet $k$ comme corps résiduel alors il existe un élément $\pi_{K}$ de $B_{K}$ relevant une uniformisante de son corps résiduel construite à l'aide de la théorie du corps des normes [F-W] telle qu'on ait encore des isomorphismes :

$$
\mathcal{E}_{K_{0}} \rightarrow B_{K}
$$

qui à la série de Laurent $\sum_{n \in \mathbb{Z}} a_{n} x^{n}$ associe l'élément $\sum_{n \in \mathbb{Z}} a_{n}\left(\pi_{K}\right)^{n}$. 


\subsubsection{L'équivalence de catégories de Fontaine}

Si $V$ est une représentation $p$-adique de $G_{K}$ on pose :

$$
D(V):=\left(B \otimes_{\mathbb{Q}_{p}} V\right)^{H_{K}} .
$$

L'action de $\varphi$ sur $B$ commute à l'action de $G_{K}$ et $D(V)$ devient un $B_{K^{-}}$ module muni d'une action de $\varphi$ qui commute avec l'action résiduelle de $\Gamma_{K}$. On obtient comme cela un $(\varphi, \Gamma)$-module étale, c'est-à-dire un $B_{K}$-module qui admet une base dans laquelle le déterminant de la matrice de $\varphi$ est une unité de l'anneau des entiers.

Réciproquement si $D$ est un $(\varphi, \Gamma)$-module on pose :

$$
V_{K}(D):=\left(B \otimes_{B_{K}} D\right)^{\varphi=1} .
$$

Fontaine a montré dans $\left[\mathrm{F}_{2}\right]$ :

Théorème 4.4.1 Le foncteur

$$
V \mapsto D(V): \underline{\operatorname{Rep}}\left(G_{K}\right) \rightarrow(\varphi, \Gamma)-\bmod \left(B_{K}, e ́ t\right)
$$

est une équivalence de catégories entre la catégorie $\operatorname{Rep}\left(G_{K}\right)$ des représentations $p$-adiques de $G_{K}$ et la catégorie $(\varphi, \Gamma)-\bmod \left(\overline{B_{K}, e ́ t}\right)$ des $(\varphi, \Gamma)$-modules étales et admet le foncteur

$$
D \mapsto V(D):(\varphi, \Gamma)-\bmod \left(B_{K}, e ́ t\right) \rightarrow \underline{\operatorname{Rep}}\left(G_{K}\right)
$$

comme quasi-inverse.

\subsubsection{L'équivalence de catégories de Cherbonnier}

Le corps $\tilde{B}$ contient le sous-corps $\tilde{B}^{\dagger}$ des éléments dits surconvergents ([Ch], [Ch-C]). Pour un entier $k$ et $x=\sum_{i \in \mathbb{Z}} p^{i}\left[x_{i}\right]$ un élément de $\tilde{B}$ on définit $w_{k}(x):=\inf _{i \leq k} v_{E}\left(x_{i}\right)$. Pour un nombre réel $r \geq 0$ on définit l'anneau $\tilde{B}_{r}^{\dagger}$ comme l'ensemble des éléments $x$ de $\tilde{B}$ tels que la suite $w_{k}(x)+\frac{p r}{p-1} k$ est bornée inférieurement. Le sous-corps $\tilde{B}^{\dagger}$ est la réunion des anneaux $\tilde{B}_{r}^{\dagger}$ pour $r \geq 0$.

On définit les anneaux $\tilde{A}^{\dagger}:=\tilde{B}^{\dagger} \cap \tilde{A}, A^{\dagger}:=\tilde{B}^{\dagger} \cap A$ et $B^{\dagger}:=\tilde{B}^{\dagger} \cap B$ et on pose :

$$
A_{K}^{\dagger}:=\left(A^{\dagger}\right)^{H_{K}}, B_{K}^{\dagger}:=\left(B^{\dagger}\right)^{H_{K}} .
$$

Le corps $B^{\dagger}$ est muni du morphisme de Frobenius $\varphi$ et d'une action du groupe de Galois $G_{K_{0}}$ et le corps $B_{K_{0}}$ est muni du morphisme de Frobenius $\varphi$ et d'une action résiduelle du quotient $G_{K_{0}} / H_{K_{0}}$. 
Plus concrètement on a l'isomorphisme canonique :

$$
\mathcal{E}_{K_{0}}^{\dagger} \rightarrow B_{K_{0}}^{\dagger}
$$

qui à la série de Laurent $\sum_{n \in \mathbb{Z}} a_{n} x^{n}$ associe l'élément $\sum_{n \in \mathbb{Z}} a_{n}\left(\pi_{K_{0}}\right)^{n}$ où $\mathcal{E}_{K_{0}}^{\dagger}$ est le sous-corps de $\mathcal{E}_{K_{0}}$ des séries de Laurent qui convergent dans une couronne $1-\epsilon<|x|<1$ pour un nombre réel $\epsilon$ non précisé.

Si $K$ est non ramifié le corps $B_{K}^{\dagger}$ est encore canoniquement isomorphe au corps $\mathcal{E}_{K}^{\dagger}$. Mais si $K$ est totalement ramifié alors il existe un élément $\pi_{K}$ de $B_{K}$ relevant une uniformisante de son corps résiduel construite à l'aide de la théorie du corps des normes $[\mathrm{F}-\mathrm{W}]$ telle qu'on ait encore des isomorphismes :

$$
\mathcal{E}_{K_{0}}^{\dagger} \rightarrow B_{K}^{\dagger}
$$

qui à la série de Laurent $\sum_{n \in \mathbb{Z}} a_{n} x^{n}$ associe l'élément $\sum_{n \in \mathbb{Z}} a_{n}\left(\pi_{K}\right)^{n}$.

Pour une représentation $p$-adique de $G_{K}$ on pose :

$$
D^{\dagger}(V):=\left(B^{\dagger} \otimes_{\mathbb{Q}_{p}} V\right)^{H_{K}}
$$

qui est un $(\varphi, \Gamma)$-module sur $B_{K}^{\dagger}$.

Réciproquement si $D$ est un $(\varphi, \Gamma)$-module on pose :

$$
V_{K}(D):=\left(B \otimes_{B_{K}} D\right)^{\varphi=1} .
$$

Le théorème suivant est conjecturé dans la Thèse de Cherbonnier [Ch] et démontré dans $[\mathrm{Ch}-\mathrm{C}]$ :

\section{Théorème 4.4.2 Le foncteur}

$$
V \mapsto D^{\dagger}(V): \underline{\operatorname{Rep}}\left(G_{K}\right) \rightarrow(\varphi, \Gamma)-\bmod \left(B_{K}^{\dagger}, e ́ t\right)
$$

est une équivalence de catégories entre la catégorie $\operatorname{Rep}\left(G_{K}\right)$ des représentations p-adiques de $G_{K}$ et la catégorie $(\varphi, \Gamma)-\bmod \left(\overline{B_{K}^{\dagger}, e ́ t}\right)$ des $(\varphi, \Gamma)$-modules étales et admet le foncteur

$$
D \mapsto V(D):(\varphi, \Gamma)-\bmod \left(B_{K}^{\dagger}, e ́ t\right) \rightarrow \underline{\operatorname{Rep}}\left(G_{K}\right)
$$

comme quasi-inverse. De plus pour une représentation p-adique $V$, on a l'isomorphisme canonique fonctoriel :

$$
D(V) \simeq B_{K} \otimes_{B_{K}^{\dagger}} D^{\dagger}(V)
$$

L'équivalence de Cherbonnier fait passer de l'anneau $B_{K}$ à l'anneau $B_{K}^{\dagger}$ plus utile du point de vue différentiel. 


\subsubsection{Le théorème de Berger}

Comme nous le verrons l'anneau qui intervient dans la théorie des équations différentielles $p$-adiques est l'anneau $\mathcal{R}_{K}(1)$ des séries de Laurent qui convergent dans une couronne $1-\epsilon<|x|<1$ pour un nombre réel $\epsilon$ non précisé. L'anneau $\mathcal{E}_{K}^{\dagger}$ est l'intersection des anneaux $\mathcal{R}_{K}(1)$ et $\mathcal{E}_{K}$.

On peut construire naturellement l'analogue galoisien de l'anneau $\mathcal{R}_{K}(1)$ que nous notons pour être cohérent $B_{K}^{a n}$ en prenant des complétés pour un topologie de la convergence uniforme sur les couronnes fermées des espaces $B_{r}^{\dagger}\left[\mathrm{B}_{1}\right]$.

Si $K$ est non ramifié on a encore un isomorphisme :

$$
\mathcal{R}_{K}(1) \rightarrow B_{K}^{a n}
$$

qui à la série de Laurent $\sum_{n \in \mathbb{Z}} a_{n} x^{n}$ associe l'élément $\sum_{n \in \mathbb{Z}} a_{n}\left(\pi_{K}\right)^{n}$ et si $K$ est totalement ramifié on a encore un isomorphisme

$$
\mathcal{R}_{K_{0}}(1) \rightarrow B_{K}^{a n}
$$

qui à la série de Laurent $\sum_{n \in \mathbb{Z}} a_{n} x^{n}$ associe l'élément $\sum_{n \in \mathbb{Z}} a_{n}\left(\pi_{K_{0}}\right)^{n}$.

L'idée nouvelle due à Fontaine $\left[\mathrm{F}_{4}\right]$ est que l'action résiduelle de $\Gamma_{K}$ permet de munir le module

$$
D^{a n}(V):=B_{K}^{a n} \otimes_{B_{K}^{\dagger}} D^{\dagger}(V)
$$

d'une connexion qui a une infinité de singularités au bord et qui commute à l'action de $\varphi$. De façon plus précise si $\gamma$ est un élément du groupe $\Gamma$ assez proche de 1 l'opérateur

$$
\Delta_{V}:=-\frac{1}{\log (\chi(\gamma))} \sum_{n \in \mathbb{N}^{*}} \frac{(1-\gamma)^{n}}{n}
$$

converge vers un endomorphisme de $D^{a n}(V)$ qui est une connexion de $B_{K}^{a n}$ modules si on munit $B_{K}^{a n}$ de l'opérateur $\Delta_{\mathbb{Q}_{p}}$. L'endomorphisme $\Delta_{V}$ ne dépend pas de l'élément $\gamma$ choisi assez proche de 1.

Par exemple pour $K_{0}$ l'opérateur $\Delta_{\mathbb{Q}_{p}}$ est égal à

$$
\log \left(1+\pi_{K_{0}}\right)\left(1+\pi_{K_{0}}\right) \frac{d}{d \pi_{K_{0}}}
$$

qui opère naturellement sur $D^{a n}(V)$. La fonction $\log \left(1+\pi_{K_{0}}\right)$ a une infinité de zéros au bord et on obtient une connexion admettant une infinité de singularités apparentes au bord. Cette idée de Fontaine particulièrement fructueuse a été mise au point par L. Berger dans sa Thèse $\left[\mathrm{B}_{1}\right]$ qui supprime ces singularités dans le cas d'une représentation de de Rham et démontre $\left[\mathrm{B}_{2}\right]$ : 
Théorème 4.4.3 Soit $K$ une extension finie de $K_{0}$ totalement ramifiée, alors il existe un foncteur:

$$
N_{d R}: \underline{\operatorname{Rep}}_{d R}\left(G_{K}\right) \rightarrow(\varphi, \Gamma, \partial)-\bmod \left(B_{K}^{a n}\right)
$$

de la catégorie $\underline{R e p}_{d R}\left(G_{K}\right)$ des représentations p-adiques de de Rham dans la catégorie $(\varphi, \Gamma, \bar{\partial})-\bmod \left(B_{K}^{a n}\right)$ des $B_{K}^{a n}(\varphi, \Gamma)$-modules munis d'une connexion non singulière qui à $V$ associe $N_{d R}(V)$. De plus la représentation $V$ est semistable sur une extension cyclotomique $K_{n}$ d'ordre $n$ de $K$ pour $n$ assez grand si et seulement si les constituants irréductibles de $N_{d R}(V)$ vu simplement comme module à connexion sont isomorphes au modules trivial $B_{K}^{a n}$.

Cette fois le foncteur $N_{d R}$ n'est pas pleinement fidèle et le passage de la représentation $p$-adique de de Rham au module différentiel associé fait perdre de l'information.

Le théorème de Berger ramène le théorème de la monodromie $p$-adique : la potentielle semi-stabilité d'une représentation $p$-adique de de Rham du groupe de Galois $G_{K}$ à construire une extension finie $K^{\prime}$ de $K$ telle que le module $N_{d R}\left(V_{K^{\prime}}\right)$ que l'obtient à partir la représentation induite du groupe de Galois $G_{K^{\prime}}$ se décompose sur l'anneau $B_{K^{\prime}}^{a n}$ construit à partir du corps résiduel de $K^{\prime}$. Ce problème relève de la théorie des équations différentielles p-adiques.

\section{La Théorie des équations différentielles $p$-adiques}

Le théorème de l'indice pour les opérateurs différentiels opérant sur des espaces de fonctions analytiques $p$-adiques a été la principale motivation dans la théorie des équations différentielles entre 1970 et 1995. Ceci a abouti à trouver la structure générale $p$-adique d'un point singulier d'un opérateur différentiel qui a permi de démontrer le théorème de l'indice en octobre 1994 pour une classe importante d'opérateurs différentiels. Cette même structure a permi de démontrer le théorème de la monodromie $p$-adique des représentations galoisiennes $p$-adiques en mai 2001.

\subsection{Le problème de l'indice}

Soit

$$
P\left(x, \frac{d}{d x}\right) \in \overline{\mathbb{Q}}\left[x, \frac{d}{d x}\right]
$$

un opérateur différentiel dont les coefficients sont des polynômes à coefficients dans le corps des nombres algébriques $\overline{\mathbb{Q}}$ sur le corps des nombres rationnels. Soit $\overline{\mathbb{Q}} \hookrightarrow K$ un plongement dans un corps $p$-adique extension complète du corps $\mathbb{Q}_{p}$ des nombres $p$-adiques. 
L'opérateur $P$ opère de façon naturelle sur l'espace $\mathcal{A}_{K}(r)$ des séries $\sum_{i \in \mathbb{N}} a_{n} x^{n}$ qui convergent pour $|x|<r$ pour un nombre réel $r>0$. Le problème est savoir si le conoyau $\mathcal{A}_{K}(r) / P \mathcal{A}_{K}(r)$ est de dimension finie sur $K$ où de façon équivalente si le conoyau $\mathcal{R}_{K}(r) / P \mathcal{R}_{K}(r)$ est de dimension finie sur $K$. C'est bien le cas si $r$ est assez petit ou assez grand, mais le cas crucial $r=1$ est encore un problème ouvert. L'importance du cas $r=1$ vient du fait que les disques ouverts de rayon 1 sont les relèvements en caractéristique zéro des points fermés en caractéristique $p>0$.

La structure $p$-adique d'un point singulier d'un opérateur différentiel permet de définir une obstruction à l'existence de l'indice qui porte sur les exposants de la monodromie. On sait démontrer que cette obstruction n'a pas lieu pour $r=1$ et les opérateurs différentiels qui proviennent de la géométrie. C'est en utilisant ce résultat qu'on a démontré que les nombres de Betti $p$-adiques d'une variété affine non singulière sur un corps de caractéristique $p$ sont finis. L'arithmétique des coefficients de l'opérateur $P\left(x, \frac{d}{d x}\right)$ intervient dans cette l'obstruction et, en général, l'indice n'existe pas si les coefficients de l'opérateurs se trouvent dans un corps $p$-adique.

\subsection{La catégorie $\operatorname{MLS}\left(\mathcal{R}_{K}(r)\right)$ des modules solubles}

Pour étudier la structure $p$-adique d'un disque singulier centré à l'origine et de rayon $r>0$ d'un opérateur différentiel il faut faire l'extension $K(x) \hookrightarrow \mathcal{R}_{K}(r)$ dans l'anneau des séries de Laurent $\sum_{n \in \mathbb{Z}} a_{n} x^{n}$ à coefficients dans $K$ et qui convergent dans une couronne $r-\epsilon<|x|<r$ pour $\epsilon>0$ non précisé. L'anneau $\mathcal{R}_{K}(r)$ n'est pas noethérien. Mais heureusement le théorème suivant $\left(\left[\mathrm{C}-\mathrm{M}_{3}, 3.1 .1\right]\right)$ qui est conséquence d'un théorème de M. Lazard permet quand même de l'étudier :

Théorème 5.2.1 Si le corps $K$ est maximalement complet l'anneau $\mathcal{R}_{K}(r)$ est de Bézout, c'est-à-dire que ses idéaux de type fini sont principaux.

En particulier l'anneau $\mathcal{R}_{K}(r)$ est cohérent : un idéal de type fini est de présentation finie. On dit qu'un corps complet ultramétrique est maximalement complet si une intersection de disques non vides emboîtés est non vide. Un corps à valuation discrète est maximalement complet, le complété d'une clôture algébrique n'est pas maximalement complet en général mais admet une extension qui est maximalement complète et algébriquement close, cf. $\left[\mathrm{C}_{1}, 1.9 .7\right]$. En utilisant ce résultat on démontre $\left(\left[\mathrm{C}-\mathrm{M}_{3}, 4.1 .2\right]\right)$ :

Théorème 5.2.2 Si le corps $K$ est maximalement complet la catégorie $\operatorname{MLC}\left(\mathcal{R}_{K}(r)\right)$ des $\mathcal{R}_{K}(r)$-modules libres de type fini muni d'une connexion est abélienne. 
Soit $\mathcal{M}$ un $\mathcal{R}_{K}(r)$-module libre de rang $m$ à connexion. Sa fonction rayon de convergence $\rho \mapsto R(\mathcal{M}, \rho)$, qui est le premier invariant de $\mathcal{M}$, est définie sur un intervalle $[r-\epsilon, r[$, pour $\epsilon>0$ assez petit par :

$$
R(\mathcal{M}, \rho):=\min \left(\rho, \liminf _{k \rightarrow \infty}\left(\left|G_{k}\right|_{\rho}\right)^{-1 / k}\right)
$$

où $G_{k}$ est la matrice de l'opérateur $\Delta^{k}:=\frac{1}{k !} \frac{d^{k}}{d x^{k}}$ dans une base. La fonction rayon de convergence ne dépend pas de la base choisie : c'est le rayon de convergence des solutions locales dans un disque générique $D\left(t_{\rho}, \rho^{-}\right)$. Un disque générique $D\left(t_{\rho}, \rho^{-}\right)$est un disque de rayon $\rho$ se trouvant au bord du disque de rayon $\rho$ dans une extension valuée assez grande $K \hookrightarrow \Omega$ telle que les points de ce disque $D\left(t_{\rho}, \rho^{-}\right)$soient transcendants sur $K$. Un disque générique existe toujours à isomorphisme près. La fonction rayon de convergence est à valeurs réelles positives et logarithmiquement concave. La limite

$$
R\left(\mathcal{M}, r^{-}\right):=\lim _{\rho \rightarrow r^{-}} R(\mathcal{M}, \rho)
$$

existe et, de plus, la fonction $R(\mathcal{M}, \rho)$ est logarithmiquement dérivable à gauche, de valeur éventuellement infini, quand $\rho \rightarrow r^{-}$. Par construction $R\left(\mathcal{M}, r^{-}\right)$est $\leq r$. On a la définition $\left(\left[\mathrm{C}^{\left.\left.-\mathrm{M}_{3}, 4.1 .1\right]\right)}\right.\right.$ :

Définition 5.2.3 On dit que $\mathcal{M}$ est soluble en $r$ si $R\left(\mathcal{M}, r^{-}\right)=r$.

On note $\operatorname{MLS}\left(\mathcal{R}_{K}(1)_{K}(r)\right)$ la catégorie des modules solubles.

Proposition 5.2.4 Si le corps $K$ est maximalement complet, la catégorie $\operatorname{MLS}\left(\mathcal{R}_{K}(r)\right)$ est une sous-catégorie pleine abélienne de la catégorie $\operatorname{MLC}\left(\mathcal{R}_{K}(r)\right)$ stable par extension, par dualité et par produit tensoriel.

Ceci résulte du théorème de Robba $\left(\left[\mathrm{R}_{1}, 4.23\right]\right)$ de l'exactitude du foncteur solution à valeurs dans l'espace des fonctions analytiques dans un disque centré en un point d'un disque générique. On en déduit que pour une suite exacte de la catégorie $\operatorname{MLC}\left(\mathcal{R}_{K}(r)\right)$ la fonction rayon de convergence du terme central est le minimum des fonctions rayons de convergence des termes extrêmes. Par ailleurs la fonction rayon de convergence est invariante par dualité.

De plus tout objet est de longueur finie et les constituants absolument irréductibles, c'est-à-dire qui restent irréductibles après toute extension finie du corps de base, sont définis après éventuelle extension finie du corps de base. 


\subsection{Le polygone de Newton en $\rho$}

Soit $\mathcal{M}$ un $\mathcal{R}_{K}(r)$-module libre de type fini à connexion défini sur une couronne $|x| \in\left[r-\epsilon, r\right.$ [ et $t_{\rho}$ un point générique au bord du disque de rayon $\rho$ pour $\rho \in[r-\epsilon, r$. En vertu du théorème de Cauchy-Lutz la dimension du $\Omega$-espace vectoriel $\operatorname{Hom}_{\mathcal{R}_{K}(r)\left[\frac{d}{d x}\right]}\left(\mathcal{M}, \mathcal{O}_{t_{\rho}}\right)$ (espace des solutions à valeurs dans l'espace des fonctions analytiques au voisinage de $t_{\rho}$ ) est égale au rang $m$ de $\mathcal{M}$. L'espace $\operatorname{Hom}_{\mathcal{R}_{K}(r)\left[\frac{d}{d x}\right]}\left(\mathcal{M}, \mathcal{O}_{t_{\rho}}\right)$ est naturellement muni d'une filtration continue $\operatorname{Hom}_{\mathcal{R}_{K}(r)\left[\frac{d}{d x}\right]}\left(\mathcal{M}, \mathcal{A}_{t_{\rho}}(s)\right)$ indexée par les nombres réels $0<s \leq \rho$. Cette filtration admet un nombre fini de sauts. Posons $s=\rho^{\beta+1}$ et si $s$ est un saut nous dirons que $\beta$ est une pente. La multiplicité $m_{\beta}$ d'une pente est par definition la dimension de l'espace vectoriel des solutions qui convergent exactement dans le disque de rayon $\rho^{\beta+1}$.

Définition 5.3.1 Le polygone de Newton $(\mathcal{M}, p, \rho)$ p-adique en $\rho$ est le polygone défini par les multiplicités $m_{\beta}$ et les produits $\beta m_{\beta}$.

Proposition 5.3.2 Le polygone de Newton $(\mathcal{M}, p, \rho)$-adique en $\rho$ est invariant par dualité et est additif dans une suite exacte courte : les multiplicités d'une pente sont additives et l'ensemble des pentes du terme du milieu est réunion des ensembles des pentes des termes extrêmes.

Ceci résulte de l'exactitude du foncteur $\mathcal{M} \rightarrow \operatorname{Hom}_{\mathcal{R}_{K}(r)\left[\frac{d}{d x}\right]}\left(\mathcal{M}, \mathcal{A}_{t_{\rho}}(s)\right)$ pour $s$ fixé. Le polygone de $N$ ewton $(\mathcal{M}, p, \rho)$ varie avec $\rho$. Il s'agit d'étudier la variation de ce polygone avec $\rho$.

\subsection{Le Théorème de la plus grande pente $p$-adique}

Théorème 5.4.1 Pour tout corps p-adique $K$, la fonction rayon de convergence $R(\mathcal{M}, \rho)$ d'un module soluble en $r$ est pour $\rho$ assez près de $r$ une fonction logarithmiquement concave, affine par morceau et admet un nombre fini de pentes qui sont des nombres rationnels positifs ou nuls de dénominateurs bornés par le rang $m$.

En particulier cette fonction est de la forme $\rho^{\beta+1}$ pour tout $\rho$ assez voisin de $r$ et pour un nombre rationnel $\beta \geq 0$.

Ce théorème est démontré dans $\left(\left[\mathrm{C}-\mathrm{M}_{3}\right], 4.2 .1\right)$. On a la définition fondamentale $\left(\left[\mathrm{C}-\mathrm{M}_{3}\right], 4.2 .2\right)$ :

Définition 5.4.2 La plus grande pente $p$-adique $\operatorname{pt}(\mathcal{M})$ de $\mathcal{M}$ est le nombre rationnel $\beta \geq 0$ défini par le théorème précédent.

On dit qu'un $\mathcal{R}_{K}(1)$-module soluble est purement de pente $\beta \geq 0$ si ses solutions locales au point générique $t_{\rho}$ en toutes un même rayon de convergence égal à $\rho^{\beta+1}$ pour tout $\rho$ assez proche de 1 . 
Le théorème précédent dit que la plus grande pente en $\rho$ d'un module soluble en $r$ est pour $\rho$ assez près de $r$ un nombre rationnel $\beta \geq 0$ indépendant de $\rho$. Nous allons voir que les autres pentes ont aussi cette propriété, mais c'est nettement plus difficile.

\subsection{Le Théorème de décomposition selon les pentes $p$-adiques et le polygone de Newton $p$-adique}

Théorème 5.5.1 Supposons que le corps de base K est maximalement complet et soit $\mathcal{M}$ un $\mathcal{R}_{K}(1)$-module soluble en $r$ appartenant au groupe des valeurs absolues du corps de base $K$, alors il admet une filtration décroissante fonctorielle stricte $\mathcal{M}_{>\gamma}$ dans la catégorie $\operatorname{MLS}\left(\mathcal{R}_{K}(1)\right)$ indexée par les nombres réels $\gamma \geq 0$ telle que les modules gradués $G r^{\gamma}(\mathcal{M})$ sont purement de pente $\gamma$ et on a la décomposition canonique et fonctorielle en somme directe :

$$
\mathcal{M} \simeq \oplus_{\gamma \geq 0} G r^{\gamma}(\mathcal{M})
$$

Théorème 5.5.2 Soit $\mathcal{M}$ un $\mathcal{R}_{K}(1)$-module de rang $m$ soluble purement de pente $\gamma>0$, alors le produit $m \gamma$ est un entier et l'indice $\chi\left(\mathcal{M}, \mathcal{R}_{K}(1)\right)$ est nul.

Ces deux théorèmes sont démontrés dans $\left[\mathrm{C}^{-\mathrm{M}_{3}}\right]$ dans le cas d'un corps lo-

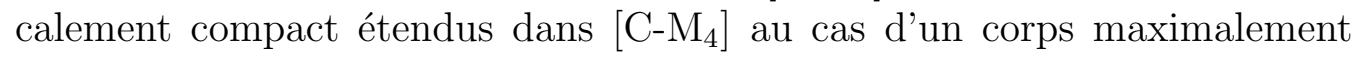
complet. Le lemme de compacité $\left(\left[\mathrm{C}-\mathrm{M}_{3}, 6.1 .2\right]\right)$ dans le cas d'un corps localement compact est généralisé en un lemme de c-compacité dans le cas d'un corps maximalement complet dans ([C-M $\left.\left.\mathrm{M}_{4}, 1.4 .2\right]\right)$. La décomposition canonique en somme directe est démontrée dans ([C- $\left.\left.\mathrm{M}_{3}, 8.3 .10\right]\right)$ sous l'hypothèse de la propriété (NL) pour le module des endomorphismes et dans ([C-M $\left.\left.\mathrm{M}_{4}, 2.4 .1\right]\right)$ dans le cas général.

Si $r$ appartient au groupe des valeurs absolues du corps en faisant le changement de variable $x \rightarrow x / a$ pour un nombre $a$ du corps $K$ de valeur absolue $r$ en se ramène à supposer que $r=1$, ce que nous ferons pour simplifier les notations et nous dirons soluble pour soluble en 1 . Le théorème 5.5.1 définit le polygone de Newton $p$-adique :

Définition 5.5.3 Supposons que le corps de base $K$ est maximalement complet, on définit le polygone de Newton p-adique Newton $(\mathcal{M}, p)$ d'un module soluble $\mathcal{M}$ par :

$$
\left(\left(m_{0}, 0\right),\left(m_{\beta_{1}}, \beta_{1} m_{\beta_{1}}\right), \ldots,\left(m_{\mathrm{pt}(\mathcal{M})}, \operatorname{pt}(\mathcal{M}) m_{\mathrm{pt}(\mathcal{M})}\right)\right) .
$$


L'entier $m_{\beta}$ est la multiplicité de la pente $\beta$, c'est-à-dire le rang du $\mathcal{R}_{K}(1)$-module $G r^{\beta}(\mathcal{M})$. Le théorème 5.5.2 montre que ses sommets sont à coordonnées entières, ce qui constitue l'analogue $p$-adique de la propriété de Hasse-Arf de la théorie $\ell$-adique. En particulier sa hauteur

$$
\operatorname{Irr}(\mathcal{M}, p):=\sum_{\beta>0} \beta m_{\beta}
$$

est un entier positif ou nul qui est nul si et seulement si le module $\mathcal{M}$ est purement de pente nulle.

Corollaire 5.5.4 Pour un module soluble en $r$, le polygone de Newton Newton $(\mathcal{M}, p, \rho)$ est indépendant de $\rho$ pour $\rho$ assez près de $r$.

En effet par construction Newton $(\mathcal{M}, p)$ coïncide avec Newton $(\mathcal{M}, p, \rho)$ pour $\rho$ assez près de $r$. Ceci montre que le polygone de Newton $p$-adique est invariant par dualité et est additif dans une suite exacte.

Pour une extension $K \rightarrow K^{\prime}$ et un module $\mathcal{M}$ notons

$$
\mathcal{M}_{K^{\prime}}:=\mathcal{R}_{K}(1)_{K^{\prime}} \otimes_{\mathcal{R}_{K}(1)} \mathcal{M}
$$

le module obtenu par changement de bases.

Proposition 5.5.5 En définissant pour tout $\gamma \geq 0$ le module $\mathcal{M}^{\leq \gamma}$ par la suite exacte

$$
0 \rightarrow \mathcal{M}_{>\gamma} \rightarrow \mathcal{M} \rightarrow \mathcal{M}^{\leq \gamma} \rightarrow 0,
$$

le module $\mathcal{M}_{>\gamma}$ est le plus grand sous-module de $\mathcal{M}$ dont toutes les pentes sont strictement plus grandes que $\gamma$ et le module $\mathcal{M} \leq \gamma$ est le plus grand quotient de $\mathcal{M}$ dont la plus grande pente est $\leq \gamma$. De plus le rang du module $\mathcal{M}^{\leq \gamma}$ est égal à la dimension sur $\Omega$ de l'espace $\operatorname{Hom}_{\mathcal{R}_{K}(r)\left[\frac{d}{d x}\right]}\left(\mathcal{M}, \mathcal{A}_{t_{\rho}}\left(\rho^{\gamma+1}\right)\right)$ des solutions de $\mathcal{M}$ à valeurs dans l'espace $\mathcal{A}_{t_{\rho}}\left(\rho^{\gamma+1}\right)$.

Ce résultat est démontré dans $\left[\mathrm{C}-\mathrm{M}_{3}\right]$.

Proposition 5.5.6 Soient $K \rightarrow K^{\prime}$ une extension de corps maximalement complets et $\mathcal{M}$ un $\mathcal{R}_{K}(1)$-module soluble, alors il existe pour tout réel $\gamma \geq 0$ un morphisme de changement de bases

$$
G r^{\gamma}\left(\mathcal{M}_{K^{\prime}}\right) \rightarrow \mathcal{R}_{K}(1)_{K^{\prime}} \otimes_{\mathcal{R}_{K}(1)_{K}} G r^{\gamma}(\mathcal{M})
$$

qui est un isomorphisme. En particulier le polygone de Newton p-adique est invariant par extension des scalaires. 
Démonstration. Comme la fonction rayon de convergence ne change pas par extension des scalaires on a alors un morphisme surjectif :

$$
\mathcal{M}_{K^{\prime}}^{\leq \gamma} \rightarrow \mathcal{R}_{K^{\prime}} \otimes_{\mathcal{R}_{K}(1)} \mathcal{M}^{\leq \gamma} \rightarrow 0
$$

Mais pour tout $\rho>0$ un disque générique $D\left(t_{\rho}, \rho^{-}\right)$au bord du disque de rayon $\rho \mathrm{du}$ corps $K^{\prime}$ est encore un disque générique $D\left(t_{\rho}, \rho^{-}\right)$au bord $\mathrm{du}$ disque de rayon $\rho$ du corps $K$. Cela entraîne en particulier que les dimensions des espaces de solutions à valeurs dans l'espace des fonctions analytiques dans le disque $D\left(t_{\rho}, \rho^{\gamma+1}\right)$ des modules $\mathcal{M}$ et $\mathcal{M}_{K^{\prime}}$ sont égales pour $\rho$ assez proche de 1 . Mais ces dimensions sont égales aux rangs des modules $\mathcal{M}_{K^{\prime}}^{\leq \gamma}, \mathcal{M} \leq \gamma$ en vertu du théorème de décomposition ([C- $\left.\left.\mathrm{M}_{3}, 6.1 .13\right]\right)$. Donc le morphisme surjectif précédent est un isomorphisme. D'autre part on a, pour $\epsilon>0$ assez petit, une suite exacte

$$
0 \rightarrow G r^{\gamma}(\mathcal{M}) \rightarrow \mathcal{M}^{\leq \gamma} \rightarrow \mathcal{M}^{\leq \gamma-\epsilon} \rightarrow 0
$$

qui montre la proposition 5.5.6.

\subsection{Le Théorème de la monodromie $p$-adique des équations diffé- rentielles}

\subsubsection{Cas de rang un}

Pour un module soluble $\mathcal{M}$ on définit la partie de pente nulle comme $\mathcal{M} \leq 0$ et la partie de pentes strictement positives comme $\mathcal{M}_{>0}$. Traditionnellement dans les théories de la ramification on désigne par partie modérée la partie de pente nulle et par partie sauvage la partie de pentes $>0$. Dans la théorie $p$-adique rien n'est moins justifié que cette terminologie, la structure de la partie modérée étant plus sauvage que la structure de la partie de pentes $>0$. Aussi nous dirons partie de pente nulle et partie de pentes $>0$ comme suggéré par les notations précédentes.

Le théorème de la monodromie $p$-adique a pour point de départ le résultat suivant dû à Robba $\left[\mathrm{R}_{4}\right]$. Soit $\mathcal{M}$ un $\mathcal{R}_{K}(1)$-module à connexion libre de rang un, la classe dans $K / \mathbb{Z}$ du résidu de la matrice de la connexion dans une base est un invariant de $\mathcal{M}$ qui ne dépend pas de la base choisie. Si $\mathcal{M}$ est soluble de pente nulle notons cet élément $\mathfrak{E x p}(\mathcal{M})$. P. Robba a montré le résultat remarquable $\left[\mathrm{R}_{4}\right]$ :

Théorème 5.6.1 Soit $\mathcal{M}$ un module de la catégorie $\operatorname{MLS}\left(\mathcal{R}_{K}(1)\right)$ de rang 1 et de pente nulle, alors $\mathcal{M}$ admet une solution non triviale de la forme $x^{-\mathfrak{E x p}(\mathcal{M})} f(x)$ où $f(x)$ est une série de $\mathcal{R}_{K}(1)$ et de plus $\mathfrak{E x p}(\mathcal{M})$ est un élément de $\mathbb{Z}_{p} / \mathbb{Z}$. 
Autrement dit si $\mathcal{M}$ est soluble de pente nulle il admet une base dans laquelle la matrice de l'action de $x \frac{d}{d x}$ est constante égale à un relèvement de l'exposant et une solution multiforme qui prolonge analytiquement les solutions locales uniformes qui ont un rayon de convergence maximum. L'existence de l'indice dans cette situation se ramène à l'existence de l'indice pour l'opérateur $x \frac{d}{d x}-\alpha$ pour un entier $p$-adique $\alpha$. Tout le problème est qu'il existe des nombres dits de Liouville pour les lesquels l'indice n'existe pas à cause du problème des petits dénominateurs. Aussi pour avoir les propriétés de finitude il faut empêcher ce phénomène.

Définition 5.6.2 Soit $\alpha$ un entier p-adique, on dit qu'il a la propriété (NL) si les séries $\sum_{i \in \mathbb{N}, \neq \alpha} \frac{x^{i}}{i-\alpha}, \sum_{i \in \mathbb{N} \neq-\alpha} \frac{x^{i}}{i+\alpha}$ ont un rayon de convergence égal 1 .

La raison d'être de la propriété (NL) est que l'indice $\chi\left(x \frac{d}{d x}-\alpha, \mathcal{R}_{K}(1)\right)$ existe si et seulement si $\alpha$ a la propriété (NL) et dans ce cas l'indice est nul. Par exemple les nombres algébriques sur $\mathbb{Q}$ ont la propriété (NL) mais dans $\left[\mathrm{R}_{1}\right] \mathrm{P}$. Robba construit des exemples d'entiers $p$-adiques qui n'ont pas la propriété (NL). D'autre part pour un opérateur d'ordre un $x \frac{d}{d x}-a(x)$ de pente nulle où $a(x)$ est une fonction de $\mathcal{R}_{K}(1)$ l'existence de l'indice ne dépend que de son exposant. Dans la théorie complexe l'exposant est bien entendu l'exposant de la monodromie.

Cette dernière interprétation géométrique suggère que, dans le cas de rang $\geq 2$, l'obstruction à l'existence de l'indice se lit dans les exposants $p$-adiques de la monodromie et tout le problème est de les définir, ce qui ne peut se faire ni à l'aide d'une base ni à l'aide du prolongement analytique. C'est sans doute là où résidait en partie l'énigme de la théorie $p$-adique qui explique son blocage.

\subsubsection{L'ensemble des exposants de rang $m$}

Aussi la situation est nettement plus compliquée. Pour tout entier $m \geq 1$ nous allons définir l'ensemble des exposants $\mathfrak{E}_{m}$ de rang $m$ comme le quotient de $\left(\mathbb{Z}_{p} / \mathbb{Z}\right)^{m} / \stackrel{\mathfrak{E}}{\sim}$ par la relation d'équivalence $\stackrel{\mathfrak{E}}{\sim}\left[\mathrm{C}-\mathrm{M}_{2}\right]$. Pour tout entier $p$-adique $\alpha$ et tout entier naturel $h$, on note $\alpha^{(h)}$ l'unique représentant entier appartenant à $\left[\frac{1-p^{h}}{2}, \frac{1+p^{h}}{2}\left[\right.\right.$ de la classe de $\alpha$ modulo $p^{h}$, et $\alpha^{(h)}:=$ $\left(\alpha_{1}^{h}, \ldots, \alpha_{m}^{h}\right)$ pour un $m$-uple $\alpha:=\left(\alpha_{1}, \ldots, \alpha_{m}\right)$. On note $\left.\right|_{\infty}$ la valeur absolue usuelle. On a la définition $\left[\mathrm{C}-\mathrm{M}_{2}\right]$ :

Définition 5.6.3 Soit $\alpha$ et $\alpha^{\prime}$ deux m-uples d'entiers $p$-adiques, on dit qu'ils sont équivalents pour la relation d'équivalence $\stackrel{\mathfrak{E}}{\sim}$ s'il existe une suite de permutations $\sigma_{h}, h \in \mathbb{N}$ de l'ensemble $\{1, \ldots, m\}$ telle que $\left|\alpha^{(h)}-\sigma_{h}\left(\alpha^{\prime}\right)^{(h)}\right|_{\infty}:=$ $\max _{i}\left|\alpha_{i}^{(h)}-\alpha_{\sigma(i)}^{\prime(h)}\right|_{\infty}$ soit un $O(h)$. 
Dans le cas $m=1$ deux entiers $p$-adiques sont équivalents si et seulement s'ils diffèrent d'un entier. La relation $\stackrel{\mathfrak{E}}{\sim}$ est asymptotique et passe au quotient par $\mathbb{Z}$, nous noterons encore $\stackrel{\mathfrak{E}}{\sim}$ la relation induite sur $\left(\mathbb{Z}_{p} / \mathbb{Z}\right)^{m}$. On a la définition $\left[\mathrm{C}-\mathrm{M}_{2}\right]$ :

Définition 5.6.4 L'ensemble $\mathfrak{E}_{m}$ des exposants de rang $m$ est l'ensemble quotient

$$
\left(\mathbb{Z}_{p} / \mathbb{Z}\right)^{m} / \stackrel{\mathfrak{E}}{\sim}
$$

On a la proposition $\left(\left[\mathrm{C}-\mathrm{M}_{2}, 5.4 .2\right]\right)$ :

Proposition 5.6.5 Soient deux entiers $n, r$ tels que $m=n+r$ alors il existe une application somme surjective $\mathfrak{E}_{n} \times \mathfrak{E}_{r} \rightarrow \mathfrak{E}_{m}$.

Cette application somme est loin d'être injective.

On note $\operatorname{Rob}\left(\mathcal{R}_{K}(1)\right)$ la sous-catégorie pleine de la catégorie $\operatorname{MLS}\left(\mathcal{R}_{K}(1)\right)$ des modules purement de pente nulle.

Théorème 5.6.6 Pour tout module $\mathcal{M}$ de rang $m$ soluble de pente nulle, on définit son exposant $\mathfrak{E x p}(\mathcal{M})$ comme un élément de l'ensemble $\mathfrak{E}_{m}$. On obtient une application exposant Exp de la catégorie $\operatorname{Rob}\left(\mathcal{R}_{K}(1)\right)$ dans la réunion des ensembles $\mathfrak{E}_{m}$. L'application exposant a les propriétés suivantes :

1) L'application exposant est additif dans une suite exacte de modules différentiels ayant la propriété de Robba l'exposant du terme médian est la somme des exposants des termes extrêmes.

2) L'exposant du dual d'un module est l'opposé de l'exposant du module.

3) La somme des éléments d'un représentant de l'exposant est indépendante comme élément de $\mathbb{Z}_{p} / \mathbb{Z}$ du représentant et est égale à l'exposant du module déterminant.

C'est une construction délicate $\left[\mathrm{C}-\mathrm{M}_{2}\right]$ qui utilise la structure de Frobenius de Christol-Dwork [C-D] mise au point à cette occasion. On a la proposition $\left[\mathrm{C}-\mathrm{M}_{2}\right]$

Proposition 5.6.7 Soit $\alpha=\left(\alpha_{1}, \ldots, \alpha_{m}\right)$ un m-uple d'entiers $p$-adiques tel que les différences $\alpha_{i}-\alpha_{j}$ ont la propriété (NL), alors pour tout $m$-uple $\alpha^{\prime}=$ $\left(\alpha_{1}^{\prime}, \ldots, \alpha_{m}^{\prime}\right)$ d'entiers $p$-adiques $\stackrel{\mathfrak{E}}{\sim}$-équivalent à $\alpha$ il existe une permutation $\sigma$ de l'ensemble $\{1, \ldots, m\}$ telle que $\alpha_{i}=\alpha_{\sigma(i)}^{\prime} \operatorname{modulo} \mathbb{Z}, i=1, \ldots, m$.

L'intérêt de la proposition précédente est que la classe d'équivalence d'un $m$-uple dont les différences ont la propriété (NL) se représente par un $m$-uple d'éléments de $\mathbb{Z}_{p} / \mathbb{Z}$ modulo une permutation. Ceci permet de définir l'ensemble de base : 
Définition 5.6.8 On définit $\mathfrak{E}_{m}^{\mathrm{NL}}$ comme le sous-ensemble de l'ensemble $\mathfrak{E}_{m}$ des exposants dont les différences ont la propriété (NL).

On peut alors considérer la sous-catégorie $\operatorname{Rob}\left(\mathcal{R}_{K}(1), \mathbf{D N L}\right)$ des modules purement de pente nulle des modules dont l'exposant a des différences qui ont la propriété (NL).

Définition 5.6.9 Pour un module $\mathcal{M}$ de la catégorie $\operatorname{Rob}\left(\mathcal{R}_{K}(1), \mathbf{D N L}\right)$, on appelle exposants p-adiques les entiers p-adiques d'un représentant de l'exposant $\mathfrak{E x p}(\mathcal{M})$, ce sont des éléments de $\mathbb{Z}_{p} / \mathbb{Z}$ bien définis non ordonnés comptés avec leur multiplicité.

On a le théorème $\left[\mathrm{C}-\mathrm{M}_{2}\right]$ :

Théorème 5.6.10 La sous-catégorie $\operatorname{Rob}\left(\mathcal{R}_{K}(1), \mathrm{DNL}\right)$ est stable par sousquotient et dans une suite exacte de cette catégorie les exposants du terme médian sont la réunion des exposants des termes extrêmes.

Prendre garde que la propriété (NL) pour les différences n'est pas stable par extension. C'est pour les objets de la catégorie $\operatorname{Rob}\left(\mathcal{R}_{K}(1)\right.$, DNL $)$ qu'on a un théorème de structure généralisant le cas de rang un. La condition (DNL) est vide en rang 1 mais hautement non triviale en rang $>1\left[\mathrm{C}-\mathrm{M}_{1}\right]$.

\subsubsection{Cas de rang $\geq 2$}

Le théorème suivant de la monodromie $p$-adique peut être considéré comme l'analogue $p$-adique du théorème de Fuchs donnant la structure des opérateurs différentiels à coefficients dans le corps des séries formelles à coefficients dans un corps de caractéristique nulle à singularité régulière :

Théorème 5.6.11 Soit $\mathcal{M}$ un $\mathcal{R}_{K}(1)$-module soluble purement de pente nulle de rang $m$ ayant la propriété (DNL) et d'exposants p-adiques $\alpha_{1}, \ldots, \alpha_{m}$, alors il admet une suite de décomposition dont les gradués sont isomorphes aux modules définis par $x \frac{d}{d x}-\alpha_{i}$.

De façon plus précise le module $\mathcal{M}$ admet une solution non triviale $x^{\alpha} c_{\alpha}(x)$ où $\alpha$ est un exposant $p$-adique et $c_{\alpha}(x)$ est une fonction de l'anneau $\mathcal{R}_{K}(1)$. Par suite l'espace vectoriel sur $K$ de ses solutions multiformes de détermination finie de la forme $\sum_{k} c_{\alpha, k}(x) x^{\alpha}(\log (x))^{k}$ est de dimension $m$ donnant un sens au prolongement multiforme des solutions locales.

De façon équivalente le module admet une base dans laquelle la matrice de l'action de $x \frac{d}{d x}$ est une matrice constante dont les valeurs propres sont égales aux exposants $p$-adiques de $\mathcal{M}$ modulo $\mathbb{Z}$.

De plus $\mathcal{M}$ admet une filtration de "monodromie" $\mathcal{M} \supset \mathcal{M}_{i}, i=0,1, \ldots$ telle que les modules gradués $\mathcal{M}_{i} / \mathcal{M}_{i+1}$ admettent des solutions bornées dans le disque générique de rayon $\rho \in[1-\epsilon, 1[$ pour $\epsilon>0$ assez petit. 
La démonstration originale de ce théorème est faite dans $\left[\mathrm{C}-\mathrm{M}_{2}\right]$ dans le cas de $\mathbb{C}_{p}$ et se descend à tout sous-corps complet de $\mathbb{C}_{p}$ par $\left(\left[\mathrm{C}-\mathrm{M}_{3}, 7.1 .2\right]\right)$. En fait cette démonstration vaut pour tout corps qui est le complété d'une clôture algébrique d'un corps à valuation discrète ainsi qu'à ses sous-corps complets. Cette condition intervient seulement dans le théorème de décomposition de Birkhoff du paragraphe 2 de $\left[\mathrm{C}-\mathrm{M}_{2}\right]$. Une variante de ce théorème due à Dwork $[\mathrm{D}]$ qui supprime la décomposition de Birkhoff vaut pour tout corps $p$-adique. Autrement dit sous la conditions (DNL) les modules irréductibles sont de rang 1 et la classe d'isomorphie est donnée par l'exposant.

Remarque 5.6.12 L'hypothèse (DNL) est minimale : on peut construire des exemples de modules de pente nulle mais n'admettant pas une base fondamentale de solutions multiformes.

Le théorème précédent donne naissance au théorème d'existence de Riemann vraiment $p$-adique à savoir que sous la condition (DNL) les fibrés $p$-adiques à connexion de pente nulle aux singularités proviennent de fibré algébrique n'ayant que des singularités régulières ayant mêmes exposants. Il permet définir la catégorie des coefficients $p$-adiques modérément ramifiés sur les courbes ayant les propriétés de finitude requises $\left(\left[\mathrm{C}-\mathrm{M}_{2}, \S 6\right]\right)$, ceci montre que c'est la bonne notion de monodromie dans la théorie $p$-adique. Les problèmes classiques liés au théorème d'existence garde un sens particulièrement naturel dans le contexte $p$-adique. En fait c'était là où résidait en partie l'énigme de la théorie $p$-adique et il n'y avait aucune chance de construire une théorie des coefficients $p$-adiques parallèle à la théorie des coefficients $\ell$-adiques sans avoir élucidé la question de la monodromie $p$-adique.

\subsection{Le théorème et la formule de l'indice}

On utilise le théorème précédent pour montrer l'existence et la nullité de l'indice :

Corollaire 5.7.1 Soit $\mathcal{M}$ un $\mathcal{R}_{K}(1)$-module soluble purement de pente 0 ayant la propriété (DNL) et dont les exposants ont la propriétés (NL), alors l'indice $\chi\left(\mathcal{M}, \mathcal{R}_{K}(1)\right)$ existe et est nul.

Dans le cas d'un corps maximalement complet, on définit l'exposant d'un module soluble comme l'exposant de sa partie de pente nulle. On dit qu'un module a la propriété (NL) si son exposant a la propriété (DNL) et si ses exposants ont la propriété (NL). Alors les théorèmes précédents entraînent le théorème de l'indice :

Corollaire 5.7.2 Supposant le corps de base maximalement complet et soit $\mathcal{M}$ un $\mathcal{R}_{K}(1)$-module soluble qui a la propriété (NL), alors l'indice $\chi\left(\mathcal{M}, \mathcal{R}_{K}(1)\right)$ existe et est nul. 
En utilisant les résultats précédents et les propriétés de l'indice généralisé $\left(\left[\mathrm{C}-\mathrm{M}_{3}, \S 8\right]\right)$ on obtient la formule de l'indice :

Théorème 5.7.3 Soient $K$ un corps maximalement complet et $P\left(x, \frac{d}{d x}\right)$ un opérateur différentiel d'ordre $m$ dont les coefficients sont des polynômes à coefficients dans le corps $K$ soluble en 1 et ayant la propriété (NL) alors l'indice $\chi\left(P, \mathcal{A}_{K}(1)\right)$ est fini et est égal à :

$$
\chi\left(P, \mathcal{A}_{K}(1)\right)=\operatorname{Irr}(P, p)+m-\operatorname{ord}_{[0,1[}\left(a_{m}\right)
$$

où ord ${ }_{[0,1}\left[\left(a_{m}\right)\right.$ est le degré du diviseur des zéros contenu dans le disque ouvert centré à l'origine et de rayon 1 du terme principal $a_{m}$ de $P$.

\subsection{Cas d'une structure de Frobenius}

On peut appliquer les théorèmes précédents pour les modules munis d'une structure de Frobenius. On suppose que le corps de base est muni d'un morphisme de Frobenius qui s'étend à toute extension finie. On fixe

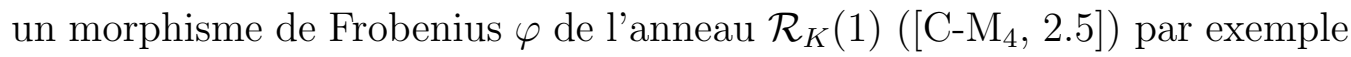
l'élévation à la puissance de $p$ de $x$ suivie du morphisme de Frobenius du corps de base $K$.

Définition 5.8.1 On définit la catégorie $\operatorname{MLS}\left(\mathcal{R}_{K}(1), \mathbf{F}\right)$ des $\mathcal{R}_{K}(1)$-modules libres de type fini à connexion $\mathcal{R}_{K}(1)$ munis d'une structure de Frobenius qui est un isomorphisme horizontal non précisé :

$$
\varphi^{h *} \mathcal{M} \simeq \mathcal{M}
$$

pour un entier $h$ non précisé.

On a $\left(\left[\mathrm{C}-\mathrm{M}_{3}, 6.3 .11\right]\right)$ :

Proposition 5.8.2 La catégorie $\operatorname{MLS}\left(\mathcal{R}_{K}(1), \mathbf{F}\right)$ est une sous-catégorie pleine de la catégorie $\operatorname{MLS}\left(\mathcal{R}_{K}(1)\right)$

Donc en vertu du théorème 5.5.1 ses objets admettent une décomposition canonique en modules admettant une seule pente. On a $\left(\left[\mathrm{C}-\mathrm{M}_{4}, 2.5 .1\right]\right)$ :

Proposition 5.8.3 Pour deux morphismes de Frobenius $\varphi, \varphi^{\prime}$ de l'anneau $\mathcal{R}_{K}(1)$ les deux catégories $\operatorname{MLS}\left(\mathcal{R}_{K}(1), \mathbf{F}\right)$ et $\operatorname{MLS}\left(\mathcal{R}_{K}(1), \mathbf{F}^{\prime}\right)$ sont égales.

La catégorie $\operatorname{MLS}\left(\mathcal{R}_{K}(1), \mathbf{F}\right)$ est stable par dualité et par produit tensoriel. Mais il n'est pas clair qu'elle soit stable par sous-quotients et par extensions. En fait c'est une conséquence du théorème de l'indice ([C- $\left.\left.\mathrm{M}_{4}, 6.0 .20\right]\right)$ : 
Théorème 5.8.4 La catégorie $\operatorname{MLS}\left(\mathcal{R}_{K}(1), \mathbf{F}\right)$ est stable par sous-quotient et extension après éventuellement extension finie du corps de base non précisée.

Mais la structure de Frobenius d'un sous-quotient n'est pas en général canoniquement induite parce qu'on ne demande pas que les morphismes horizontaux soient compatibles aux morphismes de Frobenius. On est amené à considérer la catégorie $\operatorname{MLS}\left(\mathcal{R}_{K}(1), \mathrm{pF}\right)$ des modules potentiellement munis d'une structure de Frobenius, c'est-à-dire qui admettent une structure de Frobenius après extension finie du corps de base non précisée. En vertu du théorème précédent la catégorie $\operatorname{MLS}\left(\mathcal{R}_{K}(1), \mathrm{pF}\right)$ est alors abélienne stable par extensions.

Un point de structure dans les résultats précédents est le passage des modules différentiels sur le sous-anneau $\mathcal{E}_{K}^{\dagger}$ des fonctions bornées au bord à l'anneau $\mathcal{R}_{K}(1)\left(\left[\mathrm{C}-\mathrm{M}_{3}, 6.1 .17\right]\right)$. Dans le cas d'un corps de base à valuation discrète l'anneau $\mathcal{E}_{K}^{\dagger}$ est un corps. Ce passage était déjà nécessaire

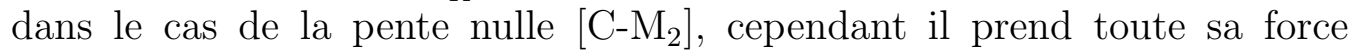

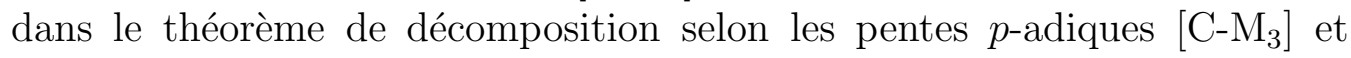
nous a obligé à développer de nouvelles méthodes pour étudier les modules différentiels sur l'anneau $\mathcal{R}_{K}(1)$ ([C-M $\left.\left.\mathrm{M}_{3}, \S \S 2,3,4,5,6\right]\right)$, en particulier l'introduction de topologie localement convexe quotient sur les modules sur les anneaux d'opérateurs différentiels et les minorations et majorations explicites très fines.

Le passage $\mathcal{E}_{K}^{\dagger} \rightarrow \mathcal{R}_{K}(1)$ a inspiré la construction d'un $\mathcal{R}_{K}(1)$-module libre muni d'une action d'un opérateur d'ordre un ayant une infinité de singularités au bord à partir d'une représentation $p$-adique du groupe de Galois $G_{K}$, mais sans singularité si la représentation est de de Rham et qui a fait le lien entre la théorie des représentations $p$-adiques en inégales caractéristiques et la théorie des équations différentielles ([Ch], [Ch-C], $\left.\left[\mathrm{B}_{1}\right],\left[\mathrm{B}_{2}\right]\right)$.

Supposons le corps de base maximalement complet et soit $\mathcal{M}$ un module à connexion muni d'une structure de Frobenius. On a $\left(\left[\mathrm{C}-\mathrm{M}_{3}, 6.3 .12\right]\right)$ :

Proposition 5.8.5 Chaque module $G r^{\gamma}(\mathcal{M})$ est muni d'une structure de Frobenius canoniquement induite par celle de $\mathcal{M}$.

En particulier la composante de pente nulle $\mathcal{M}^{\leq 0} \simeq G r^{0}(\mathcal{M})$ est munie d'une structure de Frobenius. On a alors $\left(\left[\mathrm{C}-\mathrm{M}_{2}, 5.5 .3\right]\right)$ :

Proposition 5.8.6 Les exposants p-adiques d'un module purement de pente nulle admettant une structure de Frobenius sont des nombres rationnels. 
On peut donc leur appliquer le théorème de la monodromie $p$-adique qui dit alors :

Théorème 5.8.7 Un module de pente nulle admettant une structure Frobenius se décompose en module de rang un de la forme $x \frac{d}{d x}-\alpha$ pour des nombres rationnels $\alpha$ dont le dénominateur est premier avec $p$.

C'est alors un module quasi-unipotent au sens de la théorie des équations différentielles classique.

La difficulté dans le théorème précédent pour tout rang 2 est qu'on ne peut pas définir les exposants à partir d'une base même dans le cas géométrique, ce qui nous a obligé à monter l'arsenal de l'article $\left[\mathrm{C}-\mathrm{M}_{2}\right]$. Mais c'est ce résultat fondamental et très original qui a débloqué la situation de la théorie $p$-adique des équations différentielles et ses applications.

\section{Le théorème de décomposition $p$-adique en modules de rang un}

Nous allons étendre le théorème de la monodromie au cas de pentes strictement positives. Le théorème de décomposition 5.5.1 nous réduit au cas d'une seule pente. Mais la démonstration bien qu'utilisant de façon essentielle le cas de la pente nulle et ne s'y réduit pas. Nous allons expliquer la stratégie de démonstration en renvoyant le lecteur à $\left[\mathrm{M}_{3}\right]$ pour les détails.

On note $\pi$ une solution de l'équation $\pi^{p-1}+p=0, \omega$ sa valeur absolu et on suppose que le corps $K$ contient $\pi$. Pour un intervalle $I$ de nombres réels positifs, on note $C(I)$ la couronne dont les points à valeurs dans une extension valué $K \rightarrow \Omega$ sont ceux de valeur absolue appartenant à l'intervalle $I$. Si $f$ est une fonction en la variable $x$, on note $\exp (f)$ le module de rang un à connexion défini par l'opérateur d'ordre un $\frac{d}{d x}-\frac{d}{d x}(f)$.

\subsection{Réduction au cas complètement irréductible}

Nous avons commencé l'étude de la décomposition d'un module absolument irréductible de pente strictement positive dans l'article $\left[\mathrm{C}-\mathrm{M}_{4}\right]$ en le réduisant au cas complètement irréductible par une extension finie du corps de base suivi d'une ramification d'indice premier à $p$. La conjecture 3.0.12 de cet article donne la structure d'un module complètement irréductible dont un cas particulier entraîne, comme nous allons le voir, la décomposition générale.

Définition 6.1.1 On dit qu'un module $\mathcal{M}$ de la catégorie $\operatorname{MLC}\left(\mathcal{R}_{K}(1)\right)$ est absolument irréductible s'il reste irréductible dans la catégorie $\operatorname{MLC}\left(\mathcal{R}_{K^{\prime}}(1)\right)$ pour toute extension finie $K \rightarrow K^{\prime} d u$ corps de base. 
Soit maintenant un module $\mathcal{M}$ de la catégorie $\operatorname{MLS}\left(\mathcal{R}_{K}(1)\right)$, alors son module des endomorphismes $\operatorname{End}_{\mathcal{R}_{K}(1)}(\mathcal{M})$ est encore soluble et en vertu du théorème de décomposition 5.5.1, sa partie de pente nulle $\operatorname{End}_{\mathcal{R}_{K}(1)}(\mathcal{M}) \leq 0$ est bien définie. Remarquons que celle-ci n'est jamais triviale puisqu'elle contient au moins l'endomorphisme identique. Mais, même si $\mathcal{M}$ est absolument irréductible, le rang de $\operatorname{End}_{\mathcal{R}_{K}(1)}(\mathcal{M}) \leq 0$ peut être strictement plus grand que 1. D'où la définition :

Définition 6.1.2 On dit qu'un module $\mathcal{M}$ de la catégorie $\operatorname{MLC}\left(\mathcal{R}_{K}(1)\right)$ est complètement irréductible si le rang de $\operatorname{End}_{\mathcal{R}_{K}(1)}(\mathcal{M})^{\leq 0}$ est égal à 1 .

Un module complètement irréductible est absolument irréductible. Si $\mathcal{M}$ est un module soluble l'exposant $\mathfrak{E} x p\left(\operatorname{End}_{\mathcal{R}_{K}(1)}(\mathcal{M})^{\leq 0}\right)$ est bien défini.

Définition 6.1.3 On dit qu'un module soluble $\mathcal{M}$ a la propriété (NLE) si l'exposant de la partie de pente nulle $\mathfrak{E x p}\left(\operatorname{End}_{\mathcal{R}_{K}(1)}(\mathcal{M})^{\leq 0}\right)$ a la propriété (NL).

Si $\mathcal{M}$ a la propriété $(\mathbf{N L E})$ les exposants $p$-adiques de $\mathfrak{E} x p\left(\operatorname{End}_{\mathcal{R}_{K}(1)}(\mathcal{M})^{\leq 0}\right)$ sont des éléments bien définis de $\mathbb{Z}_{p} / \mathbb{Z}$ et ils ont la propriété (NL). La condition (NLE) est stable par sous-quotient. Sous cette hypothèse fondamentale nous avons montré $\left(\left[\mathrm{C}-\mathrm{M}_{4}, 3.0 .6,3.0 .9,3.0 .10\right]\right)$ :

Théorème 6.1.4 Supposons le corps de base maximalement complet.

1) Soit $\mathcal{M}$ un $\mathcal{R}_{K}(1)$-module soluble absolument irréductible de rang $m$ ayant la propriété (NLE). Alors le rang de la partie de pente nulle $\mathcal{E}_{n d_{\mathcal{R}}}(\mathcal{M})^{\leq 0}$ est un entier $d$ divisant le rang $m$ de $\mathcal{M}$ premier avec $p$ et les exposants de $\mathcal{E} n d_{\mathcal{R}}(\mathcal{M})^{\leq 0}$ sont les classes des nombres rationnels $\left(0, \frac{1}{d}, \ldots, \frac{d-1}{d}\right)$ qui forment un groupe.

2) Soit $\mathcal{M}$ un $\mathcal{R}_{K}(1)$-module soluble absolument irréductible de rang $m$ ayant la propriété (NLE). Alors quitte à faire une extension finie du corps de base la ramification d'indice $d, x=y^{d}$ décompose $\mathcal{M}(\sqrt[d]{x})$ :

$$
\mathcal{M}(\sqrt[d]{x}) \simeq \oplus_{i=0, \ldots, d-1} \mathcal{M}_{i}
$$

en modules de rang $m / d$ complètement irréductibles et la restriction des scalaires $\mathcal{R}\left[x \frac{d}{d x}\right] \rightarrow \mathcal{R}\left[y \frac{d}{d y}\right]$ induit un isomorphisme de $\mathcal{M}$ avec l'image directe de $\mathcal{M}_{i}$ pour tout $i=0, \ldots, d-1$.

3) Soit $\mathcal{M}$ un $\mathcal{R}_{K}(1)$-module soluble de rang $m$ ayant la propriété (NLE). Alors, quitte à faire une extension finie du corps de base suivi d'une ramification d'indice e premier avec $p$, les constituants irréductibles de l'image inverse $\mathcal{M}(\sqrt[e]{x})$ sont complètement irréductibles. 
Le point clef du théorème précédent est l'existence d'un automorphisme $F$ de $\mathcal{M}$ tel que

$$
\nabla_{x \frac{d}{d x}} F=\frac{1}{d} F, \quad F^{m}=x I_{m}
$$

où $I_{m}$ est la matrice identique de rang $m\left[\mathrm{C}-\mathrm{M}_{4}\right]$. Cet automorphisme induit la décomposition après ramification d'indice $d$. Il résulte du théorème précédent qu'un module soluble absolument irréductible ayant la propriété (NLE) de pente nulle est de rang 1.

Le théorème précédent suggère la conjecture $\left(\left[\mathrm{C}-\mathrm{M}_{4}, 3.0 .12\right]\right)$ :

Conjecture 6.1.5 Un $\mathcal{R}_{K}(1)$-module soluble absolument irréductible ayant la propriété (NLE) est complètement irréductible si et seulement si son rang est une puissance positive ou nulle de $p$.

Si $m=n p^{h},(n, p)=1, h \geq 0$ est le rang d'un module $\mathcal{M}$ absolument irréductible ayant la propriété (NLE), alors la conjecture est équivalente en vertu du théorème précédent au fait que le rang $d$ de la parie de pente nulle $\operatorname{End}_{\mathcal{R}_{K}(1)}(\mathcal{M}) \leq 0$ est égale à $n$, c'est-à-dire les exposants de $\operatorname{End}_{\mathcal{R}_{K}(1)}(\mathcal{M})$ sont les nombres rationnels $(0,1 / n, \ldots,(n-1) / n)$.

\subsection{Cas d'un rang premier à $p$}

Dans l'article $\left[\mathrm{M}_{3}\right]$ nous avons démontré cette conjecture dans le cas de rang $m=n$ premier à $p$ :

Théorème 6.2.1 Soit $\mathcal{M}$ un $\mathcal{R}_{K}(1)$-module soluble absolument irréductible ayant la propriété (NLE) de rang $m$ premier à $p$ alors le rang d de la partie de pente nulle de $\operatorname{End}_{\mathcal{R}_{K}(1)}(\mathcal{M})$ est égal à $m$ et quitte à faire une extension finie $d u$ corps de base la ramification d'indice $m$ décompose le module $\mathcal{M}(\sqrt[m]{x})$ en somme directe de modules de rang 1.

Corollaire 6.2.2 Soit $\mathcal{M}$ un $\mathcal{R}_{K}(1)$-module soluble ayant la propriété (NLE) tel que ses constituants absolument irréductibles sont de rang premier à $p$, alors quitte à faire une extension finie du corps de base les constituants du module $\mathcal{M}(\sqrt[e]{x})$ sont de rang 1 où e est le ppcm des rangs des constituants absolument irréductibles de $\mathcal{M}$.

Pour démontrer le théorème de décomposition dans le cas de rang premier à $p$ on utilise les deux théorèmes suivants de nature $p$-adique $\left(\left[\mathrm{M}_{3}, 2.0 .1,3.0 .6\right]\right)$ :

Théorème 6.2.3 Soient $K$ un corps maximalement complet et algébriquement clos et $\mathcal{M}$ un $\mathcal{R}_{K}(1)$-module soluble de rang $m$ et purement de pente entière $\operatorname{pt}(\mathcal{M})=\beta=\delta p^{n}>0,(\delta, p)=1, n \geq 0$. Alors il existe un entier 
$h \geq 1$, un nombre a de $K$ de valeur absolue égale à 1 et un polynôme $\grave{a}$ coefficients dans $K$

$$
\omega_{\beta, a}(1 / x)=\frac{a}{\delta p^{n} x^{\delta p^{n}}}+\frac{b_{1}}{\delta p^{n-1} x^{\delta p^{n-1}}}+\cdots+\frac{b_{n}}{\delta x^{\delta}}
$$

tels que l'un deux cas suivants se produit :

1) Cas 1. Le module tordu $\mathcal{M} \otimes_{\mathcal{R}_{K}(1)} \exp \pi \omega_{\beta, a}\left(1 / x^{p^{h}}\right)$ est soluble et de plus grande pente strictement plus petite que $\beta$

2) Cas 2. Le module tordu $\mathcal{M} \otimes_{\mathcal{R}_{K}(1)} \exp \pi \omega_{\beta, a}\left(1 / x^{p^{h}}\right)$ est soluble et admet au moins deux pentes distinctes.

Théorème 6.2.4 Soient $K$ un corps maximalement complet et algébriquement clos et $\mathcal{M}$ un $\mathcal{R}_{K}(1)$-module soluble de rang $m$ premier avec $p$ et purement de pente fractionnaire $\operatorname{pt}(\mathcal{M})=\beta / e>0,(\beta, e)=1, e>1$, $\beta=\delta p^{n}, n \geq 0$ et $\mathcal{M}(\sqrt[e]{x})$ son image inverse par la ramification $x=y^{e}$. Alors il existe un entier $h \geq 1$, un nombre a de $K$ de valeur absolue égale $\grave{a} 1$ et un polynôme à coefficients dans $K$

$$
\omega_{\beta, a}(1 / y)=\frac{a}{\delta p^{n} y^{\delta p^{n}}}+\frac{b_{1}}{\delta p^{n-1} y^{\delta p^{n-1}}}+\cdots+\frac{b_{n}}{\delta y^{\delta}}
$$

tels et que le module tordu $\mathcal{M}(\sqrt[e]{x}) \otimes_{\mathcal{R}} \exp \pi \omega_{\beta, a}\left(1 / y^{p^{h}}\right)$ est soluble et admet au moins deux pentes distinctes.

Pour démontrer les deux théorèmes précédents on utilise la structure de Frobenius de Christol-Dwork [C-D], le théorème de Young [Y], le théorème de Robba $\left[\mathrm{R}_{4}\right]$ de l'existence d'un module soluble de rang 1 et de pente donnée à l'avance et le théorème de décomposition ([C-M $\left.\left.\mathrm{M}_{3}, 6.1 .1\right]\right)$. Les quatre résultats sont hautement non triviaux.

Sous les hypothèses du théorème 6.2.1 il s'agit de montrer que le rang de $\operatorname{End}_{\mathcal{R}_{K}(1)}(\mathcal{M})^{\leq 0}$ est égal à $m$. On peut supposer que le corps de base est maximalement complet et algébriquement clos. La torsion par un module de rang 1 ne change ni le module des endomorphismes ni l'irréductibilité absolue. Le théorème 6.2.3 nous permet de supposer que la pente est non entière, c'est-à-dire qu'on est dans les hypothèses du théorème 6.2.4 d'où l'on déduit que le rang de $\operatorname{End}_{\mathcal{R}_{K}(1)}(\mathcal{M})^{\leq 0}$ est minoré par 2. Le théorème 6.1.4 force le rang de $\operatorname{End}_{\mathcal{R}_{K}(1)}(\mathcal{M}) \leq 0$ à être exactement $m$. 


\subsection{Cas général}

Le théorème 6.2.1 a déjà de nombreuses applications $\left[\mathrm{M}_{3}\right]$, mais le plus remarquable est qu'il implique le théorème de décomposition général sans avoir à démontrer complètement la conjecture 6.1.5. Si on part d'un module soluble ayant la propriété (NLE) le théorème 6.1.4 nous ramène à supposer pour le décomposer qu'il est complètement irréductible. Le théorème 6.2.1 nous ramène au cas où le rang est divisible par $p$. L'idée consiste à décomposer le module $\operatorname{End}_{\mathcal{R}_{K}(1)}(\mathcal{M})$ en somme directe du module trivial et du module des endomorphismes de trace nulle qui est de rang premier à $p$ est d'appliquer le théorème 6.2.1 à un de ses constituants absolument irréductible de rang premier à $p$. Nous allons formuler le théorème de façon précise avant d'expliquer la stratégie de démonstration.

Notons $\mathcal{R}_{K, x}$ l'anneau $\mathcal{R}_{K}(1)$ pour pouvoir considérer des extensions $\mathcal{R}_{K, x} \rightarrow \mathcal{R}_{K^{\prime}, y}$.

Théorème 6.3.1 Supposons le corps de base à valuation discrète et considérons une sous-catégorie pleine de la catégorie $\operatorname{MLS}\left(\mathcal{R}_{K, x}\right), T U\left(\mathcal{R}_{K, x}\right)$ variable avec l'anneau $\mathcal{R}_{K, x}$ qui a les propriétés suivantes :

(i) chaque objet a la propriété (NLE),

(ii) elle est stable par dualité et produit tensoriel interne,

(iii) elle est abélienne et stable par extensions,

(iv) elle est stable par extension finie du corps de base, par ramification d'ordre premier à $p$ et par les extensions d'Artin-Schreier,

alors pour tout objet $\mathcal{M}$ de la catégorie $T U\left(\mathcal{R}_{K, x}\right)$ il existe une extension $f: \mathcal{E}_{K, x}^{\dagger} \rightarrow \mathcal{E}_{K^{\prime}, y}^{\dagger}$ composée d'un nombre fini d'extensions finies du corps de base, de ramifications d'ordre premier à $p$ et d'extensions d'Artin-Schreier telle que les constituants irréductibles de l'image inverse $f^{*} \mathcal{M}$ sont de rang 1.

$\mathrm{Vu}$ les hypothèses on se ramène au cas absolument irréductible. Soit $\mathcal{M}$ un module de la catégorie $T U\left(\mathcal{R}_{K, x}\right)$ de rang $m$ absolument irréductible. Si $m$ est premier à $p$ en vertu du théorème 6.2.1 quitte à faire une extension finie du corps de base la ramification d'indice $m$ le décompose en module de rang 1. On peut supposer que le rang $m$ est divisible par $p$. On a la décomposition de son module des endomorphismes en module scalaire et en module de trace nulle :

$$
\mathcal{E} n d_{\mathcal{R}_{K, x}}(\mathcal{M})=\mathcal{R}_{K, x} I_{m} \oplus \mathcal{E} n d_{\mathcal{R}_{K, x}}(\mathcal{M})^{t r n}
$$

qui à $u$ associe $\frac{\operatorname{tr}(u)}{m} I_{m}+u^{t r n}$. C'est une décomposition horizontale, le module des endomorphismes de trace nulle est libre de rang $m^{2}-1$ premier avec $p$ 
et admet un constituant absolument irréductible de rang premier à $p$, quitte à faire une extension finie du corps de base. Ce constituant à la propriété (NLE) vu les hypothèses $(i i)$ et $(i i i)$. En vertu du théorème 6.2.1 quitte à faire une extension finie du corps de base la ramification d'ordre le rang décompose ce constituant en modules de rang 1 . On alors supposer que le module $\mathcal{E}_{n d_{\mathcal{R}_{K, x}}}(\mathcal{M})^{t r n}$ admet un constituant de rang 1 . On utilise le résultat suivant $\left(\left[\mathrm{R}_{4}\right],[\mathrm{C}-\mathrm{C}],\left[\mathrm{C}_{2}\right],[\mathrm{Ts}]\right)$ :

Théorème 6.3.2 Soit $\mathcal{L}$ un module soluble de rang 1 , alors il existe une extension $\mathcal{E}_{K, x}^{\dagger} \rightarrow \mathcal{E}_{K, y}^{\dagger}$ composée d'extensions d'Artin-Schreier telle que le module image inverse est soluble et de pente nulle.

L'exemple type est le module $\exp \pi / x$ qui en faisant le changement de variable

$$
\frac{1}{x}=\frac{1}{y^{p}}-\frac{1}{y}
$$

devient $\exp \pi\left(\frac{1}{y^{p}}-\frac{1}{y}\right)$ qui est de pente nulle. Mais dans le cas général la situation est plus compliquée. Un module de rang 1 soluble est défini par $x^{\alpha} \exp \pi(\omega(1 / x))$ où $\omega(1 / x)$ est un polynôme en $1 / x$ et $\alpha$ un élément de $\mathbb{Z}_{p}$ définissant le résidu déterminantiel $\left[\mathrm{R}_{4}\right]$. Le module défini par $\exp \pi(\omega(1 / x))$ admet une structure de Frobenius $[\mathrm{C}-\mathrm{C}]$ qui permet de construire l'extension $f_{\omega}$ qui le trivialise $\left(\left[\mathrm{C}_{2}\right],[\mathrm{Ts}]\right)$.

Quitte à faire une extension finie du corps de base, il existe un sous $\mathcal{R}_{K, \sqrt[e]{x}}$-module libre de type fini à connexion $\mathcal{N}$ de $\mathcal{E} n d_{\mathcal{R}_{K, \sqrt[e]{x}}}(\mathcal{M})^{\operatorname{trn}}(\sqrt[e]{x})$, un polynôme $\omega(\sqrt[-e]{x})$ et un morphisme surjectif :

$$
\mathcal{N} \rightarrow x^{\alpha} \exp \pi \omega(\sqrt[-e]{x}) \rightarrow 0
$$

Le module $f_{\omega}^{*} x^{\alpha} \exp \pi \omega(\sqrt[-e]{x})$ est de pente nulle. Comme le foncteur qui à un module soluble associe sa partie de pente nulle est exact $\left(\left[\mathrm{C}-\mathrm{M}_{3}, 6.3 .1\right]\right)$, le rang de la partie de pente nulle du module $f_{\omega}^{*} \mathcal{E} n d_{\mathcal{R}_{K, x}}(\mathcal{M})^{\operatorname{trn}}(\sqrt[e]{x})$ est au moins égal à 1 . Donc le rang de la partie de pente nulle du module

$$
f_{\omega}^{*} \mathcal{E} n d_{\mathcal{R}_{K, x}}(\mathcal{M})(\sqrt[e]{x}) \simeq \mathcal{E} n d_{\mathcal{R}_{K, \sqrt[e]{x}}}\left(f_{\omega}^{*} \mathcal{M}(\sqrt[e]{x})\right)
$$

est au moins égal à 2 . Le module $f_{\omega}^{*} \mathcal{M}(\sqrt[e]{x})$ n'est pas complètement irréductible et il a encore la propriété (NLE) par hypothèse.

S'il n'est pas absolument irréductible une éventuelle extension finie du corps de base le décompose. S'il est absolument irréductible une éventuelle extension finie du corps de base suivie d'une ramification d'indice $d>1$ le décompose en vertu du théorème 6.1.4, 2). L'hypothèse de récurrence sur le rang entraîne le théorème 6.3.1. 
Le théorème précédent peut être considéré comme l'analogue $p$-adique du théorème de Turrittin et c'est un résultat de la théorie des équations différentielles $p$-adiques proprement dite.

Mais les modules $\exp \pi \omega\left(x^{-1}\right)$ de rang 1 dans la théorie $p$-adique, contrairement ceux de la théorie des équations différentielles sur le corps des séries formelles à coefficients dans un corps de caractéristique nulle, deviennent de pente nulle après une extension du type $f_{\omega}$. C'est très exactement là où se situe la différence.

Corollaire 6.3.3 Pour tout objet $\mathcal{M}$ d'une catégorie $T U(\mathcal{R})$ qui a les quatre propriétés précédentes il existe une extension $f: \mathcal{E}_{K, x}^{\dagger} \rightarrow \mathcal{E}_{K^{\prime}, y}^{\dagger}$ composée d'un nombre fini d'extensions finies du corps de base, de ramifications d'ordre premier à $p$ et d'extensions $f_{\omega}$ telle que les constituants irréductibles de l'image inverse $f^{*} \mathcal{M}$ sont de rang 1 et de pente nulle.

En effet une image inverse du type $f_{\omega}^{*}$ d'un module de rang 1 soluble de pente nulle est encore un module de rang 1 soluble de pente nulle.

Considérons la catégorie $\operatorname{MLS}\left(\mathcal{R}_{K, x}, \mathbf{F}\right)$. Elle a de façon évidente la propriété $(i i)$ et donc la propriété $(i)$. En vertu de $\left(\left[\mathrm{C}-\mathrm{M}_{4}, 2.5 .1\right]\right)$ elle a la propriété $(i v)$. Par contre elle n'a pas la propriété (iii) qui l'empêche à priori d'être tannakienne. Il faut la plonger dans la catégorie $\operatorname{MLS}\left(\mathcal{R}_{K, x}, \mathrm{pF}\right)$ des modules qui admettent une structure de Frobenius après éventuelle extension finie non précisée du corps de base, autrement dit des modules potentiellement munis d'une structure de Frobenius $\left(\left[\mathrm{C}-\mathrm{M}_{4}, 6.0 .20\right]\right)$ qui a les quatre propriétés du théorème 6.3.1. On peut donc lui appliquer le théorème 6.3.1.

Corollaire 6.3.4 Pour tout objet $\mathcal{M}$ de la catégorie $\operatorname{MLS}\left(\mathcal{R}_{K, x}, \mathrm{pF}\right)$ il existe une extension $f: \mathcal{E}_{K, x}^{\dagger} \rightarrow \mathcal{E}_{K^{\prime}, y}^{\dagger}$ composée d'un nombre fini d'extensions finies $d u$ corps de base, de ramifications d'ordre premier à $p$ et d'extensions $f_{\omega}$ telle que les constituants irréductibles de l'image inverse $f^{*} \mathcal{M}$ sont isomorphes au module trivial $\mathcal{R}_{K^{\prime}, y}$. De plus si $\mathcal{M}$ est défini sur un corps non ramifié son image inverse $f^{*} \mathcal{M}$ est aussi définie et se décompose sur un corps non ramifié.

En effet un module de rang un et de pente nulle muni d'une structure de Frobenius admet un exposant rationnel. Une ramification d'ordre premier à $p$ le trivialise sur $\mathcal{R}$. D'autre part par construction si $\mathcal{M}$ est défini sur un corps non ramifié l'image inverse $f^{*} \mathcal{M}$ est définie sur une extension non ramifiée. La décomposition qui a lieu sur une extension finie de ce corps non ramifié se descend au sous-corps non ramifié par l'argument galoisien de $\left(\left[\mathrm{C}-\mathrm{M}_{3}, 7.1 .2\right]\right)$. 
Autrement dit tout objet de la catégorie $\operatorname{MLS}\left(\mathcal{R}_{K, x}, \mathrm{p} \mathbf{F}\right)$, en particulier tout objet de la catégorie $\operatorname{MLS}\left(\mathcal{R}_{K, x}, \mathbf{F}\right)$, devient unipotent après extension composée du type précédent.

De façon plus précise la catégorie $\operatorname{MLS}\left(\mathcal{R}_{K, x}, \mathrm{p} \mathbf{F}\right)$ est tannakienne et le corollaire 6.3.4 la munit d'un foncteur fibre canonique sur une clôture algébrique $\bar{K}$, à savoir le foncteur qui à un module associe l'espace de ses solutions multiformes unipotentes de son image inverse par $f$ qui est aussi son vrai espace de ses cycles évanescents.

Les processus des théorèmes de décomposition précédents sont en quelque sorte minimaux.

Le corollaire précédent conjugué au théorème 4.4.3 entraîne le théorème 4.3.3 de la monodromie $p$-adique des représentations galoisiennes locales.

\section{L'exemple $M_{x, 1,3}$}

Nous allons illustrer le théorème de décomposition en rang 1 à l'aide de l'exemple $M_{x, 1,3}$ que nous avons étudié en détail.

Le problème de l'analogue $p$-adique du théorème de Turrittin s'est posé pour nous de façon naturelle avec les premiers contre exemples du principe de transfert pour les singularités irrégulières ([C-M $\mathrm{M}_{3}$, Introduction $\left.]\right)$. Le principe de transfert dit que sous les hypothèses de solubilité et de propriété (NL) pour les exposants, la structure $p$-adique d'un opérateur différentiel est toute pareille à sa structure en caractéristique nulle. Le théorème de transfert de Dwork dit que c'est bien le cas dans la situation d'un point ordinaire. Le théorème de transfert de Christol dit que c'est aussi le cas d'un unique point singulier régulier dans une classe résiduelle si les différences des exposants formels ont la propriété (NL).

Mais à la surprise générale des experts de l'époque le principe de transfert est faux pour une singularité irrégulière. Ceci complique considérablement la théorie des équations différentielles $p$-adiques, mais ouvre aussi un nouveau chapitre dans la théorie. L'exemple suivant, qui est un cas très particulier des équations exponentielles $M_{f, n, m}$ provenant de la géométrie et étudiées du point de vue $p$-adique dans $\left[\mathrm{M}_{1}\right]$, fournit un contre exemple non trivial au principe de transfert et donc son étude systématique, que nous avons commencé pendant l'été 1998, a été essentielle. Les équations $M_{f, n, m}$ ont été le principal catalyseur de la structure $p$-adique d'un point singulier d'une équation différentielle rappelée en partie dans la paragraphe 1 . On ne pouvait pas s'attendre à ce que leur décomposition $p$-adique soit similaire à leur décomposition formelle. 
Exemple 7.0.5 Considérons l'opérateur différentiel d'ordre 2

$$
P_{x, 1,3}\left(x, \frac{d}{d x}\right):=9 x^{3} \frac{d^{2}}{d x^{2}}+9 x^{2} \frac{d}{d x}+\frac{\pi^{2}}{3}-x .
$$

C'est un cas particulier des équations $M_{f, n, m}$ exponentielles étudiées dans $\left[\mathrm{M}_{1}\right]$. On l'obtient pour $n=1, f(x)=x, m=3$. Notons $\mathcal{M}_{x, 1,3}$ le $\mathcal{R}$-module associé, où $\mathcal{R}:=\mathcal{R}_{K}(1)$.

1) En vertu du théorème $\left(\left[\mathrm{M}_{1}, 4.1 .1\right]\right)$, pour $p \neq 3$, il est muni d'une structure de Frobenius sur l'anneau $\left(K\left[x, x^{-1}\right]\right)^{\dagger}$ comme toutes les équations exponentielles $M_{f, n, m}$ pour $p$ premier avec $m$. La hauteur $\operatorname{Irr}\left(\mathcal{M}_{x, 1,3}, \infty\right)$ de son polygone de Newton formel est égal à 1, donc la hauteur $\operatorname{Irr}\left(\mathcal{M}_{x, 1,3}, p\right)$ de son polygone de Newton $p$-adique est bornée par 1 puisqu'on a les inégalités

$$
0 \leq \operatorname{Irr}\left(\mathcal{M}_{x, 1,3}, p\right) \leq \operatorname{Irr}\left(\mathcal{M}_{x, 1,3}, \infty\right)
$$

en vertu du théorème de semi-continuité $\left(\left[\mathrm{C}-\mathrm{M}_{1}, \S 6\right]\right)$.

2) La singularité à l'infini est régulière d'exposants $1 / 3,-1 / 3$ et ses solutions $x^{1 / 3} f_{1 / 3}, x^{-1 / 3} f_{-1 / 3}$ ont un rayon de convergence borné par 1 .

Comme cet opérateur est hypergéométrique une solution de la forme $x^{\alpha} f_{\alpha}(x)$ dans $x^{\alpha} \mathcal{R}$ est nécessairement bornée $x$-adiquement à droite ou à gauche $\left(\left[\mathrm{C}-\mathrm{M}_{1}, 3.3 .7\right]\right)$. Elle ne peut pas être bornée à gauche parce que sa partie de pente nulle formelle est triviale. Si elle était bornée à droite elle serait égale à l'une des solutions $x^{1 / 3} f_{1 / 3}, x^{-1 / 3} f_{-1 / 3}$ qui ne sont pas dans $x^{ \pm 1 / 3} \mathcal{R}$. Ceci entraîne que sa partie $p$-adique de pente nulle est triviale et donc que cet opérateur est purement de pente $1 / 2$ aussi bien du point de vue formel que $p$-adique et cela pour tout $p \neq 3$. Il est alors irréductible sur l'anneau $\mathcal{R}$ pour $p \neq 3$ comme module à connexion.

3) Pour $p \neq 3$ et $p \neq 2$ on peut montrer que le groupe des exposants $p$-adiques de $\mathcal{E} n d_{\mathcal{R}}\left(\mathcal{M}_{x, 1,3}\right)$ est égal à $\{0,1 / 2\}$. Donc $\mathcal{M}_{x, 1,3}$ se décompose d'après $\left(\left[\mathrm{C}-\mathrm{M}_{4}, 3.0 .9\right]\right)$ en module de rang 1 pour $p \neq 2$ par la ramification $x=y^{2}$. Le module $\mathcal{M}_{x, 1,3}$ est absolument irréductible mais non complètement irréductible.

4) Mais pour $p=2$ le groupe des exposants du module $\mathcal{E} n d_{\mathcal{R}}\left(\mathcal{M}_{x, 1,3}\right)$ est nécessairement trivial en vertu du théorème 3.0.6 de $\left[\mathrm{C}-\mathrm{M}_{4}\right]$. Le module $\mathcal{M}_{x, 1,3}$ est complètement irréductible. En vertu de ([C- $\mathrm{M}_{3}, 6.3 .7$, 6.3.8]), en caractéristique 2 , la valuation de la pente reste égale à -1 après ramification d'ordre une puissance de 2 . En particulier le module $\mathcal{M}_{x, 1,3}$ ne se décompose pas par ramification et fournit un contre au principe de transfert provenant de la géométrie. Tout ce qui précède est explicité dans l'article ([C- $\mathrm{M}_{3}$, exemple 6.3.9]) qui a été distribué en 1995 sous forme de preprint. 
5) Avec l'aide de A. Arabia nous avons montré en 1998 que pour $p=2$ le module $\mathcal{M}_{x, 1,3}$ ne peut pas se décomposer par une suite d'extension d'ordre 2, qu'en fait sa pente reste minorée par $1 / 2$. De plus il ne provient pas d'une représentation 2-adique locale finie en égales caractéristiques 2. En fait le module $\mathcal{M}_{x, 1,3}$ ne provient pas d'une représentation $p$-adique locale finie en égales caractéristiques $p$ pour tout $p \neq 3$.

6) En caractéristique 2 le module $\mathcal{M}_{x, 1,3}$ est complètement irréductible et donc les pentes du module $\mathcal{E} n d_{\mathcal{R}}\left(\mathcal{M}_{x, 1,3}\right)^{\text {trn }}$ sont strictement positives. Ses solutions locales aux points génériques sont des sommes de produits de solutions locales du module $\mathcal{M}_{x, 1,3}$ et de solutions locales du module dual $\left(\mathcal{M}_{x, 1,3}\right)^{*}$. Donc leur rayon de convergence est minoré par le rayon de convergence des solutions locales du module $\mathcal{M}_{x, 1,3}$. La plus grande pente $\operatorname{pt}\left(\mathcal{E}_{n} d_{\mathcal{R}}\left(\mathcal{M}_{x, 1,3}\right)^{t r n}\right)$ est majorée $\operatorname{par} \operatorname{pt}\left(\mathcal{M}_{x, 1,3}\right)=1 / 2$. Le théorème de décomposition 5.5.1 montre nécessairement que $\mathcal{E} n d_{\mathcal{R}}\left(\mathcal{M}_{x, 1,3}\right)^{\text {trn }}$ est purement de pente $1 / 3$. Le passage de la pente $1 / 2$ à la pente $1 / 3$ est le point crucial qui singularise la situation $p$-adique par rapport à la situation formelle, en particulier il est absolument irréductible sur l'anneau $\mathcal{R}$, alors qu'il est réductible dans le cas formel. Dans cet exemple on voit effectivement que l'arithmétique des coefficients, imposée par la géométrie, intervient et produit des annulations qui augmentent le rayon de convergence. D'autre part cela donne un contre-exemple géométrique au principe de transfert dans le cas d'un module irréductible de rang premier à $p$.

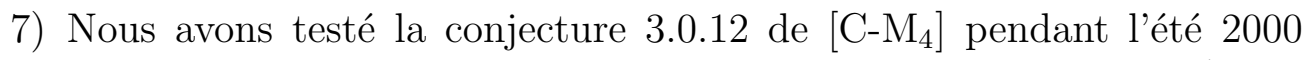
sur le module des endomorphismes de trace nulle $\mathcal{E} n d_{\mathcal{R}}\left(\mathcal{M}_{x, 1,3}\right)^{\text {trn }}$ de rang 3, qui implique la décomposition du module $\mathcal{M}_{x, 1,3}$. Le module des endomorphismes $\mathcal{E} n d_{\mathcal{R}}\left(\mathcal{E} n d_{\mathcal{R}}\left(\mathcal{M}_{x, 1,3}\right)^{\text {trn }}\right)$ est de rang 9. Montrer qu'il admet $1 / 3$ comme exposant n'est pas facile. Par contre nous avons montré que le module $\mathcal{E} n d_{\mathcal{R}}\left(\mathcal{M}_{x, 1,3}\right)^{t r n}$, par un calcul un peu compliqué parce que ce module n'est pas hypergéométrique, se décompose en rang 1 par la ramification d'indice 3, ce qui constitue une vérification de la conjecture 3.0.12 de $\left[\mathrm{C}-\mathrm{M}_{4}\right]$ et en même temps permet de suivre explicitement la décomposition du module $\mathcal{M}_{x, 1,3}$ lui-même.

Notre méthode explicite le processus de décomposition. Après une autre extension d'Artin-Schreier d'ordre 2 le module $\left.\mathcal{M}_{x, 1,3}(\sqrt[3]{x})\right)$ se décompose en rang 1. Après deux extensions successives d'Artin-Schreier d'ordre 2 il se décompose en modules de rang 1 et de pente nulle. On peut faire le calcul explicitement parce que les extensions d'ArtinSchreier sont très simples dans ce cas là. 
8) Pendant longtemps nous avons alors cherché à démontrer la conjecture 3.0.12 de $\left[\mathrm{C}-\mathrm{M}_{4}\right]$, qui rappelons-le entraîne le théorème 6.3.1, avant qu'on se rende compte que le théorème 6.2.1, qui est une vérification partielle de cette même conjecture 3.0.12 de [C-M $\left.\mathrm{M}_{4}\right]$, suffit à prouver le théorème 6.3.1.

9) La moralité de cette histoire est que le module $\mathcal{M}_{x, 1,3}$, qui ne provient pas naturellement d'une représentation 2-adique locale finie en égales caractéristiques, provient sans doute d'une représentation de de Rham en inégales caractéristiques 2 et nous laissons le soin aux experts de l'expliciter.

10) Remarquons pour terminer qu'il faut, pour cet opérateur d'ordre 2 , une extension d'ordre 6 pour le décomposer en rang 1 et d'ordre 24 pour le décomposer en rang 1 et de pente nulle, ce qui est beaucoup plus grand que dans la situation formelle. On peut montrer dans ce cas là que l'ordre de 24 est minimal. Ceci ôte tout espoir de calculer par exemple l'extension qui décompose une équation exponentielle $M_{f, n, m}$ dans le cas général.

\section{Références}

[B $\left.\mathrm{B}_{1}\right]$ Berger, L. : Représentations p-adiques et équations différentielles. Thèse de l'Université de Paris 6, 2001.

$\left[\mathrm{B}_{2}\right]$ Berger, L. : Représentations de de Rham et monodromie p-adique. Preprint daté du 14 mai 2001, 11 pages.

[Br] Brieskorn, E. : Die Monodromie der isolierten singularitäten von hyperflächen, Manuscripta Math. 2 (1970), 103-161.

[Ch] Cherbonnier, F. : Représentations p-adiques surconvergentes. Thèse de l'Université d'Orsay, 1996.

[Ch-C] Cherbonnier, F., Colmez, P- : Représentations p-adiques surconvergentes. Invent. Math. 133 (1998), 581-611.

[C-C] Chiarellotto, B., Christol, G. : Overconvergent isocrystals and Fisocrystals. Comp. Math. 100, (1996), 77-99.

$\left[\mathrm{C}_{1}\right]$ Christol, G. : Modules différentielles et Équations différentielles padiques. Queen's Paper in Pure and Applied Math. 66, Queen's University, Kingston, ON, 1983.

$\left[\mathrm{C}_{2}\right]$ Christol, G. : About a Tsuzuki theorem. In p-adic functional analysis (Ioannina, 2000), 63-74. Lecture Notes in Pure and Appl. Math. 222, Marcel Dekker, 2001.

[C-D] Christol, G., Dwork, B. : Modules différentiels sur les couronnes. Ann. Inst. Fourier 44 (1994), 663-701. 
[C-M $\mathrm{M}_{1}$ Christol, G., Mebkhout, Z. : Sur le théorème de l'indice des équations différentielles p-adiques I. Ann. Inst. Fourier 43 (1993), 1545-1574.

[C-M $\left.\mathrm{M}_{2}\right]$ Christol, G., Mebkhout, Z. : Sur le théorème de l'indice des équations différentielles p-adiques II. Ann. of Math. 146 (1997), 345-410.

[C-M $\mathrm{M}_{3}$ Christol, G., Mebkhout, Z. : Sur le théorème de l'indice des équations différentielles p-adiques III. Ann. of Math. 151 (2000), 385-457.

[C-M 4 ] Christol, G., Mebkhout, Z. : Sur le théorème de l'indice des équations différentielles $p$-adiques IV. Invent. Math. 143 (2001), 629-672.

[D] Dwork, B. : On exponents of $p$-adic differential modules. J. Reine Angew. Math. 484 (1997), 85-126.

[Fa] Faltings, G. : Crystalline cohomology and Galois p-adic representations. In Algebraic Analysis, Geometry and Number Theory (Baltimore, MD, 1988), 25-80. The John Hopkings University Press, 1989.

$\left[\mathrm{F}_{1}\right] \quad$ Fontaine, J. M. : Sur certaine types de Représentations $p$-adiques du groupe de Galois d'un corps local. Construction d'un anneau de BarsottiTate. Ann. of Math. 115 (1982), 529-577.

$\left[\mathrm{F}_{2}\right] \quad$ Fontaine, J. M. : Représentations p-adiques des corps locaux. In Grothendieck Festschrift II, 249-309. Progress in Math 87, 1991.

[F $\left.F_{3}\right]$ Fontaine, J. M. : Le corps des périodes p-adiques. Astérisque 223 (1994), 59-101.

$\left[\mathrm{F}_{4}\right] \quad$ Fontaine, J. M. : Représentations $p$-adiques semi-stables. Astérisque 223 (1994), 113-187.

$\left[\mathrm{F}_{5}\right]$ Fontaine, J.M. : Arithmétique des représentations galoisiennes $p$ adiques. Astérisque (2003) (à paraître)

[F-W] Fontaine, J. M., Winterberger, J. P. : Le corps des normes de certaines extensions algébriques de corps locaux. C. R. Acad. Sci. Paris Sér. A-B 288 (1979), no. 6, 367-370.

[F-C] Fontaine, J. M., Colmez, P. Construction des représentations $p$ adiques semi-stables. Invent. Math. 140 (2000), 1-43.

[SGA $7_{I}$ ] Grothendieck, A. : Séminaire de Géométrie Algébrique du BoisMarie 1967-68. Groupes de Monodromie en Géométrie Algébrique. Lecture Notes in Math. 288, 1972.

[G] Grothendieck, A. : Groupes de Barsotti-tate et cristaux. In Actes du Congrès Intern. Math. (Nice 1970), Tome 1, 431-436. Gauthiers-Villars, Paris, 1971.

[G-S] Grothendieck, A., Serre, J. P. : Correspondance Grothendiek-Serre. Documents Mathématiques, Société Mathématique de France, 2001.

[H-K] Hyodo, O., Kato, K. : Semi-stable reduction and crystalline cohomology with logarithmic poles. Astérisque 223 (1994), 221-268.

[K] Katz, N. : Nilpotent connexions and the Monodromy theorem : application of a result of Turrittin. Inst. Hautes Études Sci. Publ. Math. 39 (1971), 175-232. 
[Le] LÊ, D.T. : The geometry of the monodromy theorem. In C. P. Ramanujan, a Tribute, 157-173. Tata Inst. Bombay, 1978.

[Mal $]$ Malgrange, B. : Le Polynôme de Bernstein d'une singularité isolée. In Fourier integral operators and partial differential equations (Colloq. Internat., Univ. Nice, Nice, 1974), 98-119. Lecture Notes in Math. 459, Springer, Berlin, 1975.

[Ma $\mathrm{Ma}_{2}$ Malgrange, B. : Polynôme de Bernstein et cohomologie évanescente. Astérisque 101-102 (1983), 233-267.

$\left[\mathrm{M}_{1}\right]$ Mebkhout, Z. : Sur le théorème de finitude de la cohomologie $p$-adique d'une variété affine non singulière. Amer. J. Math. 119 (1997), 1027-1081.

$\left[\mathrm{M}_{2}\right]$ Mebkhout, Z. : $d R \Longrightarrow$ pst. Lettre à J. M. Fontaine datée du 28 mai 2001.

$\left[\mathrm{M}_{3}\right]$ Mebkhout, $\mathrm{Z}$. : Analogue $p$-adique du théorème de Turrittin et le théorème de la monodromie $p$-adique. Invent. Math. 148 (2002), 319-351.

[Mi] Milnor, J. : Singular Points of complexe hypersurfaces. Annals of Math. Studies 61, Princeton, N. J., 1968.

$\left[\mathrm{R}_{1}\right]$ Robba, Pн. : On the index of differential operators I. Ann. of Math. 101 (1975), 280-316.

$\left[\mathrm{R}_{4}\right]$ Robba, Pн. : Indice d'un opérateur différentiel $p$-adique IV. Cas des systèmes. Mesure de l'irrégularité dans un disque. Ann. Inst. Fourier 35 (1985), 13-55.

[S-T] Serre, J.P., TAte, J. : Good reduction of abelian varieties. Ann. of Math. 88 (1968), 492-517.

[T] Tsusi, T. : p-adic étale cohomology and crystalline cohomology in the semi-stable reduction case. Invent. Math. 137 (1999), 233-411.

[Ts] Tsuzuki, N. : Finite local monodromy of overconvergent unit-root $F$-isocrystals on a curve. Amer. J. Math. 120 (1998), 1165-1190.

[Y] Young, T. P. : Radii of convergence and index of $p$-adic differential operators. Trans. Amer. Math. Soc. 333 (1992), 769-785.

Recibido: 20 de febrero de 2002

Revisado: 7 de octubre de 2002

Zoghman Mebkhout

UFR de Mathématiques

Université de Paris 7

175 rue de Chevaleret, F-75013 Paris, France mebkhout@math.jussieu.fr

Exposé au colloque en l'honneur de J. L. Vicente, Seville, septembre 2001. 